\title{
Interference Efficiency: A New Metric to Analyze the Performance of Cognitive Radio Networks
}

\author{
Mohammad Robat Mili and Leila Musavian, Member, IEEE
}

\begin{abstract}
In this paper, we develop and analyze a novel performance metric, called interference efficiency, which shows the number of transmitted bits per unit of interference energy imposed on the primary users (PUs) in an underlay cognitive radio network (CRN). Specifically, we develop a framework to maximize the interference efficiency of a CRN with multiple secondary users (SUs) while satisfying target constraints on the average interference power, total transmit power, and minimum ergodic rate for the SUs. In doing so, we formulate a multiobjective optimization problem (MOP) that aims to maximize ergodic sum rate of SUs and to minimize average interference power on the primary receiver. We solve the MOP by first transferring it into a single objective problem (SOP) using a weighted sum method. Considering different scenarios in terms of channel state information (CSI) availability to the SU transmitter, we investigate the effect of CSI on the performance and power allocation of the SUs. When full CSI is available, the formulated SOP is nonconvex and is solved using augmented penalty method (also known as the method of multiplier). When only statistical information of the channel gains between the $\mathrm{SU}$ transmitters and the PU receiver is available, the SOP is solved using Lagrangian optimization. Numerical results are conducted to corroborate our theoretical analysis.
\end{abstract}

Index Terms-Underlay cognitive radio networks, interference efficiency, multiobjective optimization, full and limited channel state information.

\section{INTRODUCTION}

$\mathbf{T}$ HE scarcity of spectrum resources is one of the major challenges for enabling future generations of wireless communication systems. In contrast, several statistical studies and measurements on the spectrum utilization, carried-out by the federal communications commission (FCC), have shown that a significant portion of the spectrum is temporally and geographically under-utilized although limited portions, which are mainly related to unlicensed parts of the spectrum, are overloaded. For example, amateur radios and paging networks are under-utilized whereas cellular systems are highly overloaded [1]. Cognitive radio (CR) technologies target the

Manuscript received October 1, 2015; revised February 6, 2016, June 13, 2016, and November 2, 2016; accepted December 8, 2016. Date of publication January 2, 2017; date of current version April 7, 2017. This work was supported by the UK EPSRC under Grant EP/N032268/1. Part of this paper is submitted for possible publication at ICC 2017. The associate editor coordinating the review of this paper and approving it for publication was J.-M. (Jerry) Park.

M. R. Mili is with the School of Electrical Engineering, Sharif University of Technology, Tehran 11365-8639, Iran (e-mail: mohammad.robatmili@ieee.org).

L. Musavian is with the School of Computer Science and Electronics Engineering, University of Essex, Colchester CO4 3SQ, U.K. (email: leila.musavian@essex.ac.uk).

Digital Object Identifier 10.1109/TWC.2016.2647252 spectrum scarcity challenge by promising a flexible spectrum utilization management such that spectrum resources are shared intelligently across different networks of licensed and non-licensed users. The key principle of CRNs is to improve efficiency and flexibility in spectrum usage by allowing nonlicensed users, referred to as secondary users (SUs), to access the resources owned by licensed users, referred to as primary users (PUs), in an opportunistic manner. Intelligent spectrum sharing, is a particular case of CR technologies that is also referred to by underlay CRN. In this technology, the spectrum sharing scenario where SUs in CRNs are allowed to coexist with the PUs as long as the PUs' quality-of-service (QoS) is not harmfully affected by the SU network existence is practiced. The SUs have the obligation to intelligently utilize their resources and adapt to their surrounding environment such that the interference they induce to the PUs is less than a predefined threshold level [2]-[4].

Despite the traditional wireless networks that are noise limited, the future generation CRNs are affected significantly by interference [5]-[8]. In [6], distributed energy efficient discrete spectrum sharing strategy selection is investigated in cognitive MIMO interference channels with limited feedback while using a game theoretic framework. In order to reduce interference and save energy, a mechanism for shutting down links is also offered in [6]. Traditionally, spectrum efficiency (SE), that shows the number of bits per spectrum unit utilization, has been considered as a designing performance metric [8]. However, such metric does not necessarily reflect the performance of the network in terms of the interference limitations. In general, the aims of the recent research studies in underlay CRNs have been focused on maximizing the CRN throughput subject to imposing constraint on the level of the interference imposed on the PU receivers. We further note that most of the research literature in spectrum sharing systems has focused on maximizing the ergodic capacity of a single user CRN coexisting with a primary link, while less focus has been given to analyzing the effects of multiple secondary links on the performance on either CR or PU networks.

In this trend, the ergodic and outage capacity offered by a dynamic spectrum sharing approach in a single-antenna fading primary network has been investigated under different interference power constraints in [9]-[11]. These constraints at the PU receiver belong to the long-term constraint, regulating the average interference power, and short-term constraint, limiting the instantaneous interference power across all the fading states, while assuming that perfect channel side information (CSI) is available at both the receiver and the transmitter of the SU. 
The SU transmitter is offered a feedback path between its transmitter and the PU receiver to get the CSI [12] and [13]. In [14], the effect of different levels of side information on the ergodic capacity of a simple spectrum sharing scenario is investigated. A step forward to analyze the performance of CRNs in a more practical setting, namely, considering imperfect or limited CSI is considered in [15]-[17] where the ergodic capacity of a CR user with imperfect channel information has been investigated. We note that in the abovementioned works, the interference from the PU transmitter to the $\mathrm{SU}$ receiver is ignored, or approximated, and the capacity is evaluated based on the signal-to-noise-ratio (SNR).

On the other hand, we note that wireless communication systems mostly require more than one QoS requirements to be maintained [19]-[21]. These QoSs are usually maintained by formulating and solving the resource allocation problem as MOPs. For example, in [19], a generalized resource allocation optimization problem for the downlink transmission with guaranteed QoS as an MOP is formulated. MOPs focus on the simultaneous optimization of two or more conflicting objective functions where the most preferred optimal solution needs to be chosen in the presence of trade-offs between objectives [22]. MOPs are considered widely in recent literature (e.g. [23]-[28]). In detail, considering multicarrier systems, [23] proposed an algorithm to jointly maximize the throughput and minimize the transmission power while satisfying target constraints on the bit-error-rate (BER) per subcarrier and on the total transmit power. An MOP approach to maximize the $\mathrm{CR}$ system throughput and minimize its transmit power of orthogonal frequency division multiplexing (OFDM)-based CR systems in spectrum sensing mode while guaranteeing a maximum BER, transmit power budget, and a maximum number of allocated bits per subcarrier was considered in [24]. A MOP for the design of a Pareto optimal resource allocation algorithm which incorporates three important system parameters, namely, total transmit power minimization, energy harvesting efficiency maximization, and interference power leakage-to-transmit power ratio minimization is formulated in [27]. In [28], a MOP was formulated to improve the energy efficiency and then solved by first transferring it into a single objective problem (SOP), namely, a power minimization problem, by using the $\varepsilon$-constraint method.

Although, in most of previously mentioned works, the interference is taken into account by including an interference constraint in the system problem formulation, but given the increasing importance and effect of interference on the performance of spectrum sharing systems, a more elaborate study is required to analyze the system performance in terms of the imposed interference power on neighbouring users. In this paper, we introduce a novel performance metric called interference efficiency (IE) which shows the number of bits transmitted per unit of interference energy imposed on the PU receivers. We formulate the system optimization program to maximize the IE of the SU network and solve it using an MOP approach. Specifically, we maximize the ergodic sum rate of multiple SUs, which is the numerator of the IE formulation, and minimize the interference power imposed on the PUs, which is the denominator of the IE.
The main contribution of this paper are:

- We introduce a novel performance metric for CRNs, i.e., IE, and optimize the system performance in a multi-user underlay CRN.

- In contrast to most previous works which assumed single secondary link sharing the same channel with one primary link, we here consider a model where multiple SUs coexists with one PU. Under this system model, we formulate the system optimization problem, which is to maximize IE subject to average transmit and interference power constraints.

- We solve the formulated IE-optimization problem through an MOP that jointly maximizes the ergodic sum rate of multiple SUs and minimizes the interference power on the PU receivers from the SU transmitters while satisfying target constraints on the average interference power, total SU transmit power and minimum ergodic rates for the SUs. We then prove a Lemma that shows the Pareto Optimal region of the proposed MOP is inclusive of the IE-maximization problem solution.

- In order to investigate the effect of different levels of CSI availability on the power allocation and performance of the CR users, various MOPs are presented with difference levels of CSI at SU transmitter. We consider cases with full CSI and with only limited CSI. We note that having a full CSI when there are multiple SUs in the system, can be very challenging and can impose overhead on the system. By studying both scenarios, we are able to find practical solutions for when a full CSI is not available, and we also are able to analyse what performance penalty we face due to reduced CSI available at the SU transmitter. Closed-form results for evaluating the maximum ergodic sum rate are derived over Rayleigh fading channels for most cases.

- Via numerical results, we analyze the IE of the CRN and investigate the trade-off between contradicting objectives that are the maximum sum rate against the minimum average interference power.

The rest of this paper is organized as follows. Section II describes the system model. Section III proposes new MOP for multiple secondary links under full and partial CSI available at the SU transmitters. Interference Acquisition problem is investigated in Section IV. Numerical results are presented in section V and finally Section VI concludes the paper.

\section{SySTEM MODEL}

In this paper, we assume multiple secondary users sharing spectrum with a single point-to-point PU. All the considered nodes can access the spectrum simultaneously, as long as the total interference power imposed on the primary receiver is limited below a certain threshold. The network of the secondary users are assumed to be multiple secondary transmitters communicating simultaneously with their respective receivers over the same spectrum. As shown in Fig. 1, a spectrum sharing scenario is assumed where a CR network with multiple secondary links ( $K$ links) shares the same channels with an existing primary link. 


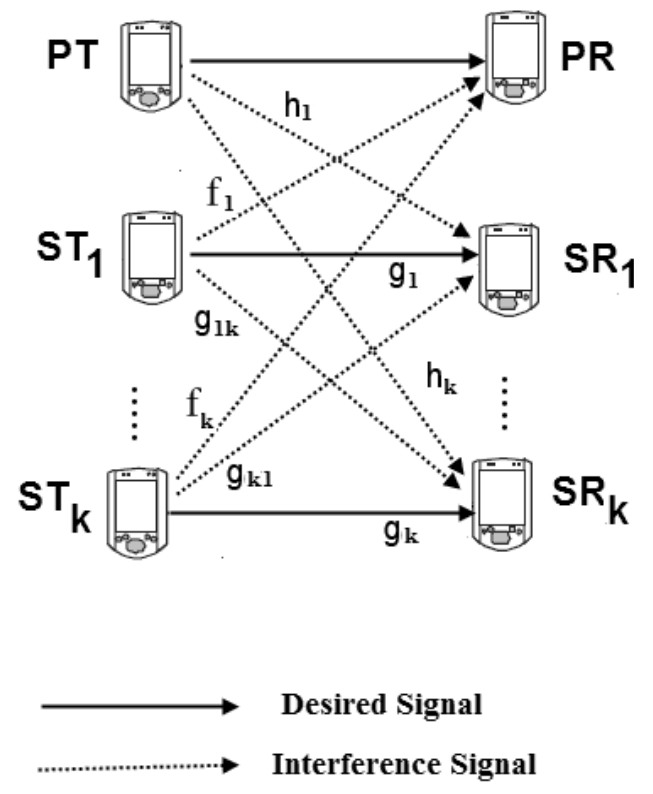

Fig. 1. Cognitive radio with multiple secondary links.

The link between the $k$ th $\mathrm{SU}$ transmitter and the $k$ th $\mathrm{SU}$ receiver is characterized by its instantaneous channel power gain $g_{k}$ and an additive white Gaussian noise (AWGN) $n_{k}$. The noise $n_{k}$ is an independent random variable with the distribution $C N\left(0, \sigma_{n}^{2}\right)$ (circularly symmetric complex Gaussian variable with mean zero and variance $\sigma_{n}^{2}$ ).

We assume that the PU transmits with a fixed transmission power $\rho$. The channel between the PU transmitter and the $k$ th SU receiver is denoted by $h_{k}$. The instantaneous received signal-to-interference-plus-noise ratio (SINR) at the $k$ th SU receiver is given by

$$
\operatorname{SINR}_{k}=\frac{P_{k} g_{k}}{N_{0}+\rho h_{k}+\sum_{j=1, j \neq k}^{K} P_{j} g_{j k}},
$$

where $N_{0}=\sigma_{n}^{2} B, P_{k}$ refers to the instantaneous power of the $k$ th SU transmitter ${ }^{1}, g_{j k}$ represents an interference channel gain between the $k$ th $\mathrm{SU}$ receiver and other SU transmitters. We note that the effect of interference among the SUs is taken into consideration in SINR formulation in (1). All the channel fading are considered to be independent and identically distributed (i.i.d.) Rayleigh flat-fading channels with unit variance. The channel power gains are assumed to remain constant during one fading block and change to independent values in the following fading block.

We further assume that the PU imposes an interference constraint on the total interference power received on its receiver as a result of the CRN operation. Specifically, the total average interference power imposed by the transmission of the CR users on the PU receivers is assumed to be below a predefined interference threshold, $Q_{\text {avg }}$. The interference

\footnotetext{
${ }^{1}$ We note that the transmit power of the $\mathrm{SU}$ is a function of the available CSI at the SU transmitter. Since we will consider various kinds of CSI availability at the SU transmitter, here we refer to this power in general by $P_{k}$. A more detailed presentation of the SU transmission power will be provided in each section based on the available CSI at the SU transmitter.
}

power constraint, hence, can be formulated as

$$
\sum_{k=1}^{K} \mathbb{E}\left[P_{k} f_{k}\right] \leq Q_{\mathrm{avg}}
$$

where $\mathbb{E}[$.$] denotes the statistical expectation and f_{k}$ represents the channel power gain between the $k$ th SU transmitter and the PU receiver.

In this paper, we consider interference efficiency as a system performance metric and define it as the number of bits transmitted per unit of interference energy imposed on the PU receiver. The IE can hence be formulated as

$$
\eta_{\mathrm{IE}}=\frac{\sum_{k=1}^{K} \mathbb{E}\left[\ln \left(1+\mathrm{SINR}_{k}\right)\right]}{\sum_{k=1}^{K} \mathbb{E}\left[P_{k} f_{k}\right]} .
$$

The importance of the introduced concept of IE lays in the fact that in spectrum sharing channels in general, and in CRNs in particular, the major constraint that limits the system performance is the constraint on the imposed interference power on the neighboring primary receivers rather than the SUs' transmit power constraints. One main approach for investigating the efficiency against a limited resource in communication systems is to find the ratio of the desirable metric (here, the achievable rate) and the limited resource. We refer to the SE when the efficiency is calculated as a ratio of the rate and the occupied bandwidth, when available bandwidth is limited. On the other hand, in Green radio communication networks, wherein the energy consumption is the major limiting factor for the system, the concept of energy efficiency (EE) that calculates the number of communicated bits versus the consumed energy, and is defined as the ratio between the achievable rate and the consumed energy, is being considered widely as the defining performance metric.

An information theoretic analysis of channels with imposed received power constraints, rather than traditionally considered transmit power constraints, due to the fact that the dominant constraint in some systems, e.g., in interference channels or CRNs, will be the interference power constraint (rather than the transmit power constraint) was provided first in [18]. This motivates the new concept of the IE that calculates the number of communicated bits per unit of imposed interference energy of the neighbouring receivers. Once the performance metric of the system is formulated according to the proposed IE concept, the resources can be managed and optimally allocated to fit best with the characteristics of the interference limited systems. This can be achieved by maximizing the number of transmitted bits versus the unit of imposed interference energy, hence maximizing the IE. With considering only SE as the performance metric, the resources will be allocated to maximize the rate, with or without considering a fixed target for the interference; henceforth a dynamic allocation of resources for maximizing the performance per unit of interference cannot be achieved.

As mentioned before, in this paper, we consider a single channel single-cell CRN. We note that for a single-cell scenario, the case for multiple channels can be easily obtained from a single channel case that have been considered in this paper. The is due to the fact that the multichannel problem 
will be a summation of the convex functions, hence yielding a convex optimization problem. In multi-cell multi-channel systems, on the other hand, the interference for the channels that are used in difference cells need to be taken into account, and hence the denominator of the IE formulation will be converted into a summation of interferences over difference channels and from difference cells. A survey on resource allocation techniques in multichannel CRNs is given in [3].

\section{IE OPTIMIZATION AND MOP FORMULATION}

Here, we present the problem formulation considered in this paper. As previously stated, we consider IE as the system performance metric and aim to design a power allocation strategy that maximizes the IE of a CRN with $K$ SUs. The problem formulation can hence be given as

$$
\begin{aligned}
\max _{P_{k}} & \eta_{\mathrm{IE}} \\
\text { s.t. } & \sum_{k=1}^{K} \mathbb{E}\left[P_{k} f_{k}\right] \leq Q_{\mathrm{avg}}, \\
& \sum_{k=1}^{K} \mathbb{E}\left[P_{k}\right] \leq P_{\mathrm{avg}}, \\
& \mathbb{E}\left[\ln \left(1+\mathrm{SINR}_{k}\right)\right] \geq R_{\mathrm{avg}}, \quad \text { for } k=1, \ldots, K,
\end{aligned}
$$

where $P_{\text {avg }}$ indicates the maximum total power of the SU transmitters and $R_{\text {avg }}$ is the minimum ergodic capacity of the each SU link. The objective function in (4a) is fractional non-convex optimization problem. Here, we propose to solve this optimization problem using an MOP approach such that we simultaneously maximize the numerator and minimize the denominator of (4a). In detail, we formulate an MOP that jointly maximizes the ergodic sum rate of multiple secondary links in the $\mathrm{CR}$ networks and minimizes the total average interference power on the PU recevier, when considering full or partial CSI scenarios. The MOP can, hence, be formulated as

$$
\begin{aligned}
\max _{P_{k}} & \sum_{k=1}^{K} \mathbb{E}\left[\ln \left(1+\operatorname{SINR}_{k}\right)\right], \\
\min _{P_{k}} & \sum_{k=1}^{K} \mathbb{E}\left[P_{k} f_{k}\right], \\
\text { s.t. } & \sum_{k=1}^{K} \mathbb{E}\left[P_{k} f_{k}\right] \leq Q_{\mathrm{avg}}, \\
& \sum_{k=1}^{K} \mathbb{E}\left[P_{k}\right] \leq P_{\mathrm{avg}}, \\
& \mathbb{E}\left[\ln \left(1+\operatorname{SINR}_{k}\right)\right] \geq R_{\mathrm{avg}}, \quad \text { for } k=1, \ldots, K .
\end{aligned}
$$

In the following, we prove that the Pareto optimal region of the introduced MOP includes the solution for the fractional IE-maximization problem.

Lemma 1: The solution to the MOP given in (5a)-(5e) covers the solution of the IE-maximization problem introduced in (4a)-(4d).

Proof: The proof is given in Appendix A.
Now, we use a well-known technique to solve the MOP, which is to linearly combine the normalized competing objective functions into a single objective function, through weighting coefficients that reflect the required preferences [22]. We note that to combine the two MOP's competing objectives, namely, the ergodic sum rate and total average interference power objectives, we normalize them to their respective reference values so that they become unitless. For the convenience of notation, however, the normalization factors are not presented in the following formulations and solutions. Hence, the objective functions in the MOP (5) can be changed into

$\max _{P_{k}} v\left(\sum_{k=1}^{K} \mathbb{E}\left[\ln \left(1+\operatorname{SINR}_{k}\right)\right]\right)-(1-v)\left(\sum_{k=1}^{K} \mathbb{E}\left[P_{k} f_{k}\right]\right)$,

where $v$ denotes the weighting coefficient which is between 0 and 1 indicating the importance of different objectives.

\section{A. Full CSI $\left[P_{k}\left(g_{k}, f_{k}, h_{k}, g_{1 k}, g_{2 k}, \ldots, g_{K k}\right)\right]$}

In this section, we assume that full CSI is available to the SU transmitter. Henceforth, the transmission power of the $k$ th SU is a function of full CSI and is represented by $P_{k}\left(g_{k}, f_{k}, h_{k}, g_{1 k}, g_{2 k}, \ldots, g_{K k}\right)$. However, for the ease of notation, in this section we refer to $P_{k}\left(g_{k}, f_{k}, h_{k}, g_{1 k}, g_{2 k}, \ldots, g_{K k}\right)$ by $P_{k}^{\text {full. }}$. The objective function in this section, hence, is according to (6), only replacing $P_{k}$ with $P_{k}^{\text {full }}$.

Since the objective function (6) under full CSI is a noncovex function [29], we here offer a method known as augmented Lagrange to deal with the nonconvex optimization (6) subject to (5c)-(5e). In nonconvex optimization problem, non-zero duality gap between the primal problem and its dual may occur, which prevents ordinary Lagrangian duality from giving the optimal solution. This method augments the Lagrangian function to eliminate these duality gap [30], [32], [33]. The "augmentation" which consists of a penalty-like quadratic term is introduced into the objective function in order to "convexify" the problem.

In an optimization problem with general inequality constraints, this method, which is a combination of penalty function and local duality methods, converts the inequality constraints into equality constraints by introducing squared additional variables. The augmented Lagrangian differs from the standard Lagrangian because of the squared terms, while it differs from the quadratic penalty function in having the summation term including Lagrangian multipliers. In this sense, it is a combination of the Lagrangian function and the quadratic penalty function. This method eliminates the constraints and adds them to the cost function as a penalty term giving a high cost to infeasible points. Berteskas in [30] has shown that the augmented Lagrangian is locally convex when penalty parameter becomes sufficiently large. This algorithm reduces the possibility of ill conditioning by introducing explicit Lagrange multiplier estimates into the function to be minimized, which is known as the augmented Lagrangian function. In contrast 
to the penalty functions, the augmented Lagrangian function largely preserves smoothness and no longer requires the penalty parameter to go to infinity for the method to converge. Augmented Lagrangian algorithms are based on successive minimization of the augmented Lagrangian function in which the multiplier estimates and penalty parameter are held fixed in each iteration and then updated between iterations [30], [31]. This method has been used in [28] to obtain the minimum transmission power giving the maximum energy efficiency of a CRNs.

The augmented Lagrangian function can be expressed as

$$
\begin{aligned}
& L_{\sigma}\left(P_{k}^{\text {full }}, \mu, \lambda\right) \\
& =v\left(\sum_{k=1}^{K} \mathbb{E}\left[\ln \left(1+\operatorname{SINR}_{k}\right)\right]\right)-(1-v)\left(\sum_{k=1}^{K} \mathbb{E}\left[P_{k}^{\text {full }} f_{k}\right]\right) \\
& +\frac{1}{2 \sigma}\left[\left(\left(\left[\lambda+\sigma\left(\sum_{k=1}^{K} \mathbb{E}\left[P_{k}^{\text {full }} f_{k}\right]-Q_{\text {avg }}\right)\right]^{+}\right)^{2}-\lambda^{2}\right)\right. \\
& +\left(\left(\left[\mu+\sigma\left(\sum_{k=1}^{K} \mathbb{E}\left[P_{k}^{\text {full }}\right]-P_{\text {avg }}\right)\right]^{+}\right)^{2}-\mu^{2}\right) \\
& \left.\quad+\sum_{k=1}^{K}\left(\left(\left[\xi_{k}+\sigma\left(R_{\text {avg }}-\mathbb{E}\left[\ln \left(1+\operatorname{SINR}_{k}\right)\right]\right)\right]^{+}\right)^{2}-\xi_{k}^{2}\right)\right],
\end{aligned}
$$

where $\lambda, \mu$ and $\xi=\left[\xi_{1}, \xi_{2}, \ldots, \xi_{K}\right]^{T}$ are Lagrangian dual variables, $\sigma$ is an adjustable penalty parameter and [.] $]^{+}$stands for $\max \{0, \cdot\}$. We note that the penalty term corresponding to the inequality constraints is continuously differentiable in $P_{k}^{\text {full }}$. Hence, the problem in (7) can be solved by an iterative algorithm to update $\lambda, \mu, \xi$ and $\sigma$ until the convergence criteria is met. In this method, the following iterations for $\lambda, \mu$ and $\xi$ are implemented as:

$$
\begin{aligned}
& \lambda^{(n+1)}=\left[\lambda^{(n)}+\sigma\left(\sum_{k=1}^{K} \mathbb{E}\left[P_{k}^{\text {full }} f_{k}^{(n)}\right]-Q_{\text {avg }}\right)\right]^{+}, \\
& \mu^{(n+1)}=\left[\mu^{(n)}+\sigma\left(\sum_{k=1}^{K} \mathbb{E}\left[P_{k}^{\text {full }}\right]-P_{\text {avg }}\right)\right]^{+}, \\
& \xi_{k}^{(n+1)}=\left[\xi_{k}^{(n)}+\left(R_{\mathrm{avg}}-\mathbb{E}\left[\ln \left(1+\operatorname{SINR}_{k}\right)\right]\right)\right]^{+},
\end{aligned}
$$

where $P_{k}^{\text {full }}{ }^{(n)}, \lambda^{(n)}, \mu^{(n)}$ and $\xi_{k}^{(n)}$ are the values of $P_{k}^{\text {full }}, \lambda$, $\mu$ and $\xi_{k}$ at stage $n$, respectively. The steps for the power control algorithm are shown in Table 1 , in which $P_{k}^{\text {full }}{ }^{(n+1)}$, $\lambda^{(n+1)}, \mu^{(n+1)}$ and $\xi_{k}^{(n+1)}$ are updated to maximize ergodic sum rate.

In this method, the rate of convergence changes by increasing $\sigma$ such that large value of $\sigma$ brings fast convergence rate but it may bring computational difficulty to minimizing the augmented Lagrangian. The linear rate of convergence of the augmented Lagrangian method is given in [32] and [33] where
TABLE I

PROPOSED ALGORITHM

1) Initially set $n=0, \sigma^{(n)}, P_{k}^{\text {full }}(n), \lambda^{(n)}, \mu^{(n)}$ and $\xi_{k}^{(n)}$.

2) Perform unconstrained optimization on the augmented Lagrangian function

$$
\begin{aligned}
& L_{\sigma^{(n)}}\left(P_{k}^{\text {full }^{(n)}}, \lambda^{(n)}, \mu^{(n)}, \xi_{k}^{(n)}\right) \text { in (7) } \\
& \text { to get } P_{k}^{\text {full }}{ }^{(n+1)} .
\end{aligned}
$$

3) set $\lambda^{(n+1)}$ by (8).

4) set $\mu^{(n+1)}$ by (9).

5) set $\xi_{k}^{(n+1)}$ by (10).

6) set $\sigma^{(n)}, n=n+1$.

7) Check the convergence criteria

if $\left\|P_{k}^{\text {full }^{(n)}}-P_{k}^{\text {full }}{ }^{(n+1)}\right\|<\epsilon$, where $\epsilon$ is

a given small constant then stop.

Otherwise, set $P_{k}^{\text {full }}{ }^{(n+1)}=P_{k}^{\text {full }^{(n)}}$ and

return to Step 2.

nonconvex nonlinear optimization problems are constrained by inequality relations. The convergence rate of this method is linear, whose ratio constant is proportional to $1 / \sigma$, when the penalty parameter $\sigma$ exceeds a threshold $\bar{\sigma}>0$. The significance of the result in [33, Ch. 3] is due to the fact that theoretically we can choose a large $\sigma$ to accelerate the convergence, which partially demonstrates why the performance of this method has been practically high. Another issue significantly affecting the convergence rate is to choose a quadratic penalty function. Hence, the convergence rate can become sublinear or superlinear if a different penalty function is taken. Bertsekas in [30] has suggested increasing $\sigma$ gradually to a certain threshold value.

\section{B. Partial CSI}

In order to discuss the significance of having knowledge of instantaneous $g_{k}, f_{k}, h_{k}$ and $\left(g_{1 k}, g_{2 k}, \ldots, g_{K k}\right)$ at the $k$ th $\mathrm{SU}$ transmitter, the power allocation at SU transmitter is evaluated under different cases in terms of what kind of knowledge about these channel gains are available at the SU transmitters. In the first case, we assume that only a statistical information about $\left(g_{1 k}, g_{2 k}, \ldots, g_{K k}\right)$ is available at the $k$ th SU transmitter. In other word, the SU is not provided with instantaneous knowledge of $\left(g_{1 k}, g_{2 k}, \ldots, g_{K k}\right)$. Henceforth, the $k$ th SU power becomes a function of $g_{k}, f_{k}$ and $h_{k}$ which is represented by $P_{k}\left(g_{k}, f_{k}, h_{k}\right)$. In the following cases, we reduce the level of CSI of $g_{k}, f_{k}$ and $h_{k}$ available at the $k$ th SU transmitter to discuss the significance of having these channel gains on the performance of the $k$ th SU. In the following, we will explain these scenarios in more detail.

1) Knowledge of $g_{k}, f_{k}$ and $h_{k}\left[P_{k}\left(g_{k}, f_{k}, h_{k}\right)\right]$ : Here, we assume only partial information about $\left(g_{1 k}, g_{2 k}, \ldots, g_{K k}\right)$, namely, the statistical channel knowledge, is available at the $k$ th SU transmitter, therefore the $k$ th SU transmit power becomes a function of only $g_{k}, f_{k}$ and $h_{k}$. In such case, the 
MOP can be modified as

$$
\begin{aligned}
& \max _{P_{k}} \sum_{k=1}^{K} \mathbb{E}[\ln (1 \\
& \left.\left.+\frac{P_{k}\left(g_{k}, f_{k}, h_{k}\right) g_{k}}{N_{0}+\rho h_{k}+\mathbb{E}\left[\sum_{j=1, j \neq k}^{K} P_{j}\left(g_{j}, f_{j}, h_{j}\right) g_{j k}\right]}\right)\right], \\
& \min _{P_{k}} \sum_{k=1}^{K} \mathbb{E}\left[P_{k}\left(g_{k}, f_{k}, h_{k}\right) f_{k}\right], \\
& \text { s.t. } \sum_{k=1}^{K} \mathbb{E}\left[P_{k}\left(g_{k}, f_{k}, h_{k}\right) f_{k}\right] \leq Q_{\mathrm{avg}}, \\
& \sum_{k=1}^{K} \mathbb{E}\left[P_{k}\left(g_{k}, f_{k}, h_{k}\right)\right] \leq P_{\mathrm{avg}}, \\
& \mathbb{E}[\ln (1 \\
& \left.\left.+\frac{P_{k}\left(g_{k}, f_{k}, h_{k}\right) g_{k}}{N_{0}+\rho h_{k}+\mathbb{E}\left[\sum_{j=1, j \neq k}^{K} P_{j}\left(g_{j}, f_{j}, h_{j}\right) g_{j k}\right]}\right)\right] \\
& \geq R_{\mathrm{avg}}, \quad \text { for } k=1, \ldots, K \text {, }
\end{aligned}
$$

which is a convex optimization program. The objectives in the above MOP becomes

$$
\begin{array}{rl}
\max _{P_{k}} & v\left(\sum_{k=1}^{K} \mathbb{E}[\ln (1\right. \\
+ & \left.\left.\left.\frac{P_{k}\left(g_{k}, f_{k}, h_{k}\right) g_{k}}{N_{0}+\rho h_{k}+\mathbb{E}\left[\sum_{j=1, j \neq k}^{K} P_{j}\left(g_{j}, f_{j}, h_{j}\right) g_{j k}\right]}\right)\right]\right) \\
& -(1-v)\left(\sum_{k=1}^{K} \mathbb{E}\left[P_{k}\left(g_{k}, f_{k}, h_{k}\right) f_{k}\right]\right) .
\end{array}
$$

By applying the expectation on the interference power from other SU transmitters in the denominator of expression (11a), this interference term becomes a constant value. Hence, according to the definition of the convex function (the Hessian is positive definite), (12) converts into a convex function. The convex structure can guarantee that the primal and dual problem have the same solution so we form the Lagrangian dual problem and solve the dual problem [34]. We employ the
Lagrangian approach for the convex optimization problem (12) subject to (11c)-(11e) by forming the Lagrangian function and taking the derivative of the Lagrangian function with respect to $P_{k}\left(g_{k}, f_{k}, h_{k}\right)$. Then, letting the derivative equal to zero gives the optimal transmission power $P_{k}\left(g_{k}, f_{k}, h_{k}\right)$ as

$$
P_{k}\left(g_{k}, f_{k}, h_{k}\right)=\left[\frac{v+\xi_{k}}{(1-v+\lambda) f_{k}+\mu}-\frac{A+\rho h_{k}}{g_{k}}\right]^{+},
$$

where $A=N_{0}+\mathbb{E}\left[\sum_{j=1, j \neq k}^{K} P_{j} g_{j k}\right]$. Note that the optimum power allocation $P_{k}\left(g_{k}, f_{k}, h_{k}\right)$, the instantaneous power at the SU transmitter, is only a function of $g_{k}, f_{k}$ and $h_{k}$, and average value of all interference from other SU transmitters which are constant values. In (13) by considering the constraint $P_{k}\left(g_{k}, f_{k}, h_{k}\right) \geq 0$, we get

$$
g_{k} \geq \frac{A+\rho h_{k}}{v+\xi_{k}}\left((1-v+\lambda) f_{k}+\mu\right) .
$$

In order to account for the maximum ergodic sum rate and the minimum interference power, the following iteration search based on the sub-gradient method can be implemented while substituting the obtained power (13) in constraints (11c)(11e) as equation (15)-(17) (shown at the bottom of this page) where $\alpha$ is a positive gradient search step-size. In theory, when the step-size $\alpha$ is small enough this approach converges to a definite number [35], [36]. A detailed explanations on how (15)-(17) can be obtained are given in Appendix B. Then, we can get the maximum sum rate under Rayleigh fading by substituting (13) into (11a) and using [37] as equation (18) (shown at the top of next page) where $C$ is the achieved ergodic capacity and $\mathrm{Ei}($.$) is the exponential integral function$ defined as $\operatorname{Ei}(x)=\int_{-\infty}^{x} \frac{e^{t}}{t} d t$. (18) is a closed-form expression for the ergodic sum rate of the secondary links when the $k$-th SU transmitter has access to the instantaneous channel gains of $g_{k}, f_{k}$ and $h_{k}$.

2) Knowledge of $f_{k}$ and $h_{k}\left[P_{k}\left(f_{k}, h_{k}\right)\right]$ : In order to find the impact of having the knowledge of instantaneous CSI of $g_{k}$ at the SU transmitter, here the maximum sum rate with only having statistical information of $g_{k}$ at the $k$ th SU transmitter is computed and compared with the results of the previous sections. We note that since it is easier to obtain the knowledge of the direct channel gains than the cross channel gains, this case is only investigated to discuss the significance of having $g_{k}$ at the SU transmitter and to compare this case with the other considered scenarios. In this scenario, the MOP (11),

$$
\begin{aligned}
& \lambda^{(n+1)}=\left[\lambda^{(n)}+\alpha\left(\sum_{k=1}^{K} \mathbb{E}\left[\left(\frac{v+\xi_{k}^{(n)}}{\left(1-v+\lambda^{(n)}\right) f_{k}+\mu^{(n)}}-\frac{A+\rho h_{k}}{g_{k}}\right) f_{k}\right]-Q_{\mathrm{avg}}\right)\right]^{+}, \\
& \mu^{(n+1)}=\left[\mu^{(n)}+\alpha\left(\sum_{k=1}^{K} \mathbb{E}\left[\left(\frac{v+\xi_{k}^{(n)}}{\left(1-v+\lambda^{(n)}\right) f_{k}+\mu^{(n)}}-\frac{A+\rho h_{k}}{g_{k}}\right)\right]-P_{\mathrm{avg}}\right)\right]^{+}, \\
& \xi_{k}^{(n+1)}=\left[\xi_{k}^{(n)}+\alpha\left(R_{\mathrm{avg}}-\mathbb{E}\left[\ln \left(\frac{g_{k}\left(v+\xi_{k}^{(n)}\right)}{\left(A+\rho h_{k}\right)\left(\left(1-v+\lambda^{(n)}\right) f_{k}+\mu^{(n)}\right)}\right)\right]\right)\right]^{+},
\end{aligned}
$$




$$
\begin{aligned}
C= & \sum_{k=1}^{K} \int_{0}^{\infty} \int_{0}^{\infty} \int_{\frac{A+\rho h_{k}}{v+\xi_{k}^{(n+1)}}\left(\left(1-v+\lambda^{(n+1)}\right) f_{k}+\mu^{(n+1)}\right)}^{\infty} \ln \left(\frac{g_{k}}{\rho h_{k}+A} \frac{v+\xi_{k}^{(n+1)}}{\left(1-v+\lambda^{(n+1)}\right) f_{k}+\mu^{(n+1)}}\right) \\
& \times e^{-f_{k}-h_{k}-g_{k}} d g_{k} d f_{k} d h_{k}=K\left(-\operatorname{Ei}\left(-\frac{A \mu^{(n+1)}}{v+\xi_{k}^{(n+1)}}\right)+e^{\frac{A}{\rho}} \operatorname{Ei}\left(-\frac{A\left(\mu^{(n+1)} \rho+\xi_{k}^{(n+1)}+v\right)}{\rho\left(v+\xi_{k}^{(n+1)}\right)}\right)\right. \\
& -e^{\frac{A}{\rho}+\frac{\mu^{(n+1)} \rho+v+\xi_{k}^{(n+1)}}{\rho\left(1-v+\lambda^{(n+1)}\right)}} \operatorname{Ei}\left(-\frac{\left(A\left(1-v+\lambda^{(n+1)}\right)+v+\xi_{k}^{(n+1)}\right)\left(\mu^{(n+1)} \rho+v+\xi_{k}^{(n+1)}\right)}{\left(1-v+\lambda^{(n+1)}\right) \rho\left(v+\xi_{k}^{(n+1)}\right)}\right) \\
& \left.+e^{\frac{\mu^{(n+1)}}{1-v+\lambda^{(n+1)}}} \operatorname{Ei}\left(\mu^{(n+1)}\left(-\frac{1}{1-v+\lambda^{(n+1)}}-\frac{A}{v+\xi_{k}^{(n+1)}}\right)\right)\right)
\end{aligned}
$$

can be update as follows:

$$
\begin{aligned}
& \max _{P_{k} \geq 0} \sum_{k=1}^{K} \mathbb{E}\left[\int_{0}^{\infty} \ln (1\right. \\
& \left.\left.+\frac{P_{k}\left(f_{k}, h_{k}\right) g_{k}}{N_{0}+\rho h_{k}+\mathbb{E}\left[\sum_{j=1, j \neq k}^{K} P_{j}\left(f_{j}, h_{j}\right) g_{j k}\right]}\right) e^{-g_{k}} d g_{k}\right] \\
& \min _{P_{k}} \sum_{k=1}^{K} \mathbb{E}\left[P_{k}\left(f_{k}, h_{k}\right) f_{k}\right] \\
& \text { s.t. } \sum_{k=1}^{K} \mathbb{E}\left[P_{k}\left(f_{k}, h_{k}\right) f_{k}\right] \leq Q_{\text {avg }} \text {, } \\
& \sum_{k=1}^{K} \mathbb{E}\left[P_{k}\left(f_{k}, h_{k}\right)\right] \leq P_{\mathrm{avg}}, \\
& \mathbb{E}[\ln (1 \\
& \left.\left.+\frac{P_{k}\left(f_{k}, h_{k}\right) g_{k}}{N_{0}+\rho h_{k}+\mathbb{E}\left[\sum_{j=1, j \neq k}^{K} P_{j}\left(f_{j}, h_{j}\right) g_{j k}\right]}\right)\right] \\
& \geq R_{\text {avg }} \text {, for } k=1, \ldots, K \text {, }
\end{aligned}
$$

where the expectation is with respect to the channel gains $f_{k}$ and $h_{k}$. Notice that (19a) and (19b) subject to (19c )-(19e) is mathematically equivalent to the following problem

$$
\begin{array}{rl}
\max _{P_{k}^{\prime} \geq 0} & v\left(\sum_{k=1}^{K} \mathbb{E}[\ln (1\right. \\
& \left.\left.\left.+\frac{P_{k}^{\prime}\left(g_{k}, f_{k}, h_{k}\right) g_{k}}{N_{0}+\rho h_{k}+\mathbb{E}\left[\sum_{j=1, j \neq k}^{K} P_{j}\left(f_{j}, h_{j}\right) g_{j k}\right]}\right)\right]\right) \\
& -(1-v)\left(\sum_{k=1}^{K} \mathbb{E}\left[P_{k}^{\prime}\left(g_{k}, f_{k}, h_{k}\right) f_{k}\right]\right) \\
\text { s.t. } & \sum_{k=1}^{K} \mathbb{E}\left[P_{k}^{\prime}\left(g_{k}, f_{k}, h_{k}\right) f_{k}\right] \leq Q_{\text {avg }}
\end{array}
$$

$$
\begin{aligned}
& \sum_{k=1}^{K} \mathbb{E}\left[P_{k}^{\prime}\left(g_{k}, f_{k}, h_{k}\right)\right] \leq P_{\mathrm{avg}}, \\
& \mathbb{E}[\ln (1 \\
& \left.\left.+\frac{P_{k}^{\prime}\left(g_{k}, f_{k}, h_{k}\right) g_{k}}{N_{0}+\rho h_{k}+\mathbb{E}\left[\sum_{j=1, j \neq k}^{K} P_{j}\left(f_{j}, h_{j}\right) g_{j k}\right]}\right)\right] \\
& \geq R_{\text {avg }}, \quad \text { for } k=1, \ldots, K,
\end{aligned}
$$

where the expectation is with respect to the channel gains $g_{k}, f_{k}$ and $h_{k}$. Here, we regarded the integration in (19a) with respect to $g_{k}$ as an expectation with respect to a dummy random variable $g_{k}$, which is distributed with exponential distribution. $P_{k}^{\prime}\left(g_{k}, f_{k}, h_{k}\right)$ can also be regarded a dummy power allocation which is a function of $g_{k}, f_{k}$ and $h_{k}$.

Following the same procedure by applying the Lagrangian approach, we find $P_{k}^{\prime}\left(g_{k}, f_{k}, h_{k}\right)$ as

$$
P_{k}^{\prime}\left(g_{k}, f_{k}, h_{k}\right)=\left[\frac{v+\xi_{k}}{(1-v+\lambda) f_{k}+\mu}-\frac{\rho h_{k}+A}{g_{k}}\right]^{+}
$$

where $A=N_{0}+\mathbb{E}\left[\sum_{j=1, j \neq k}^{K} P_{j} g_{j k}\right]$. Then, we can find the optimal power allocation, $P_{k}\left(f_{k}, h_{k}\right)$, as the average of $P_{k}^{\prime}\left(g_{k}, f_{k}, h_{k}\right)$ over $g_{k}$ as equation (22)

$$
\begin{aligned}
& P_{k}\left(f_{k}, h_{k}\right) \\
& =\int_{\frac{\left((1-v+\lambda) f_{k}+\mu\right)\left(\rho h_{k}+A\right)}{v+\xi_{k}}}^{\infty}\left(\frac{v+\xi_{k}}{(1-v+\lambda) f_{k}+\mu}-\frac{\rho h_{k}+A}{g_{k}}\right) \\
& \quad \times e^{-g_{k} d g_{k}} \\
& =\frac{e^{-\frac{\left(\rho h_{k}+A\right)\left((1-v+\lambda) f_{k}+\mu\right)}{v+\xi_{k}}}\left(v+\xi_{k}\right)}{(1-v+\lambda) f_{k}+\mu} \\
& \quad+\left(\rho h_{k}+A\right) \operatorname{Ei}\left(-\frac{\left(\rho h_{k}+A\right)\left((1-v+\lambda) f_{k}+\mu\right)}{v+\xi_{k}}\right)
\end{aligned}
$$

which gives the power at the $k$-th SU transmitter when only CSI of $f_{k}$ and $h_{k}$ are available at the $k$-th SU transmitter.

Similar iteration search-based on the sub-gradient method as indicated in (15)-(17) should be implemented to obtain the maximum ergodic sum rate by substituting (22) into (19a) 
which can be simplified into

$$
\begin{aligned}
C= & \sum_{k=1}^{K} \int_{0}^{\infty} \int_{0}^{\infty}\left(-e^{\frac{B}{e^{-B}+B \operatorname{Ei}(-B)}}\right. \\
& \times \operatorname{Ei}\left(-B-\frac{B}{e^{-B}+B \operatorname{Ei}(-B)}\right) \\
& \left.+e^{-B} \ln \left(1+e^{-B}+B \operatorname{Ei}(-B)\right)\right) \\
& \times e^{-f_{k}} e^{-h_{k}} d f_{k} d h_{k},
\end{aligned}
$$

where $B=\frac{\left(\rho h_{k}+A\right)\left(\left(1-v+\lambda^{(n+1)}\right) f_{k}+\mu^{(n+1)}\right)}{v+\xi_{k}^{(n+1)}}$. (23) is an expression for the ergodic sum rate of the secondary links when only instantaneous channel gains of $f_{k}$ and $h_{k}$ are provided at the transmitter of the $k$-th SU. We observe that closed-form expressions are not obtainable for (23), and hence, we need to solve the equation numerically.

3) Knowledge of $g_{k}$ and $h_{k}\left[P\left(g_{k}, h_{k}\right)\right]$ : Here, the maximum of the ergodic sum rate with a reduced side information of $f_{k}$ at the $k$ th $\mathrm{SU}$ transmitter is computed. Therefore, we disregard the effect of $f_{k}$ from the power allocation, resulting in the following optimization problem

$$
\begin{aligned}
& \max _{P_{k} \geq 0} \sum_{k=1}^{K} \mathbb{E}[\ln (1 \\
& \left.\left.+\frac{P_{k}\left(g_{k}, h_{k}\right) g_{k}}{N_{0}+\rho h_{k}+\mathbb{E}\left[\sum_{j=1, j \neq k}^{K} P_{j}\left(g_{j}, h_{j}\right) g_{j k}\right]}\right)\right] \\
& \min _{P_{k}} \sum_{k=1}^{K} \mathbb{E}\left[P_{k}\left(g_{k}, h_{k}\right) f_{k}\right] \\
& \text { s.t. } \sum_{k=1}^{K} \mathbb{E}\left[P_{k}\left(g_{k}, h_{k}\right) f_{k}\right] \leq Q_{\mathrm{avg}} \\
& \sum_{k=1}^{K} \mathbb{E}\left[P_{k}\left(g_{k}, h_{k}\right)\right] \leq P_{\mathrm{avg}}, \\
& \mathbb{E}[\ln (1 \\
& \left.\left.+\frac{P_{k}\left(g_{k}, h_{k}\right) g_{k}}{N_{0}+\rho h_{k}+\mathbb{E}\left[\sum_{j=1, j \neq k}^{K} P_{j}\left(g_{j}, h_{j}\right) g_{j k}\right]}\right)\right] \\
& \geq R_{\mathrm{avg}}, \quad \text { for } k=1, \ldots, K,
\end{aligned}
$$

which $(24 \mathrm{c})$ can be simplified into

$$
\sum_{k=1}^{K} \mathbb{E}\left[P_{k}\left(g_{k}, h_{k}\right)\right] \leq Q_{\text {avg }}
$$

Similarly, by applying the Lagrangian approach, we get the optimal power allocation as

$$
P_{k}\left(g_{k}, h_{k}\right)=\left[\frac{v+\xi_{k}}{1-v+\lambda+\mu}-\frac{\rho h_{k}+A}{g_{k}}\right]^{+},
$$

where $A=N_{0}+\mathbb{E}\left[\sum_{j=1, j \neq k}^{K} P_{j}\left(g_{j}, h_{j}\right) g_{j k}\right]$.
Again, we use a similar iteration search based on the subgradient method as shown in equations (15)-(17) to get the maximum ergodic sum rate by substituting (26) in (24a) and using [37] as

$$
\begin{aligned}
C= & K\left(-\operatorname{Ei}\left(-\frac{1-v+\lambda^{(n+1)}+\mu^{(n+1)}}{v+\xi_{k}^{(n+1)}} A\right)\right. \\
& \left.+e^{\frac{A}{\rho}} \operatorname{Ei}\left(-\frac{A+\left(\frac{1-v+\lambda^{(n+1)}+\mu^{(n+1)}}{v+\xi_{k}^{(n+1)}}\right) \rho A}{\rho}\right)\right),
\end{aligned}
$$

where (27) is a closed-form expression for the ergodic sum rate of the SU links when only instantaneous channel gains $g_{k}$ and $h_{k}$ are provided at the transmitter of the $k$ th SU.

4) Knowledge of $g_{k}$ and $f_{k}\left[P\left(g_{k}, f_{k}\right)\right]$ : Here, we find the sum rate with a reduced side information when instantaneous knowledge of $h_{k}$ is not made available at the $k$ th SU transmitter. Hence, by disregarding $h_{k}$, the power of SU transmitter depends on $g_{k}$ and $f_{k}$. The MOP (11) hence is modified as

$$
\begin{aligned}
& \max _{P_{k} \geq 0} \sum_{k=1}^{K} \mathbb{E}\left[\int_{0}^{\infty} \ln (1\right. \\
& \left.+\frac{P_{k}\left(g_{k}, f_{k}\right) g_{k}}{N_{0}+\rho h_{k}+\mathbb{E}\left[\sum_{j=1, j \neq k}^{K} P_{j}\left(g_{j}, f_{j}\right) g_{j k}\right]}\right) \\
& \left.\times e^{-h_{k}} d h_{k}\right] \\
& \min _{P_{k}} \sum_{k=1}^{K} \mathbb{E}\left[P_{k}\left(g_{k}, f_{k}\right) f_{k}\right] \\
& \text { s.t. } \sum_{k=1}^{K} \mathbb{E}\left[P_{k}\left(g_{k}, f_{k}\right) f_{k}\right] \leq Q_{\mathrm{avg}} \text {, } \\
& \sum_{k=1}^{K} \mathbb{E}\left[P_{k}\left(g_{k}, f_{k}\right)\right] \leq P_{\text {avg }}, \\
& \mathbb{E}\left[\int_{0}^{\infty} \ln (1\right. \\
& \left.+\frac{P_{k}\left(g_{k}, f_{k}\right) g_{k}}{N_{0}+\rho h_{k}+\mathbb{E}\left[\sum_{j=1, j \neq k}^{K} P_{j}\left(g_{j}, f_{j}\right) g_{j k}\right]}\right) \\
& \left.\times e^{-h_{k}} d h_{k}\right] \geq R_{\mathrm{avg}}, \quad \text { for } k=1, \ldots, K,
\end{aligned}
$$

where the expectation is with respect to the channel gains $g_{k}$ and $f_{k}$. The optimal power allocation in the optimization problem (28a) and (28b) subject to (28c)-(28e) is given by

$$
P_{k}\left(g_{k}, f_{k}\right)=\left[\frac{v+\xi_{k}}{(1-v+\lambda) f_{k}+\mu}-\frac{\rho+A}{g_{k}}\right]^{+}
$$

where $A=N_{0}+\mathbb{E}\left[\sum_{j=1, j \neq k}^{K} P_{j}\left(g_{j}, f_{j}\right) g_{j k}\right]$.

Similarly, the iteration search based on the sub-gradient method can be used and the maximum ergodic sum rate by 
substituting (29) in (28a) is expressed as

$$
\begin{aligned}
C= & -K \operatorname{Ei}\left(-\frac{\rho+A}{v+\xi_{k}^{(n+1)}} \mu^{(n+1)}\right)+K e^{\frac{\mu^{(n+1)}}{1-v+\lambda^{(n+1)}}} \\
& \times \operatorname{Ei}\left(-\frac{\lambda^{(n+1)}+\frac{\rho+A}{v+\xi_{k}^{(n+1)}}\left(1-v+\lambda^{(n+1)}\right) \lambda^{(n+1)}}{1-v+\lambda^{(n+1)}}\right) .
\end{aligned}
$$

The expression given in (30) represents a closed-form solution for the ergodic sum rate of the secondary links when only CSI of $g_{k}$ and $f_{k}$ are available at the $k$-th SU transmitter.

5) No Knowledge of CSI [Constant P]: Here, we assume that no instantaneous CSI is available at the $k$ th SU transmitter, therefore, the best strategy for the SU is to transmit with a constant power. Hence, the MOP simplifies into

$$
\begin{aligned}
& \max _{P_{k} \geq 0} \sum_{k=1}^{K} \mathbb{E}\left[\int_{0}^{\infty} \int_{0}^{\infty} \ln (1\right. \\
& \left.+\frac{P_{k} g_{k}}{N_{0}+\rho h_{k}+\mathbb{E}\left[\sum_{j=1, j \neq k}^{K} P_{j} g_{j k}\right]}\right) \\
& \left.\times e^{-g_{k}} e^{-h_{k}} d g_{k} d h_{k}\right] \\
& \min _{P_{k}} \sum_{k=1}^{K} \mathbb{E}\left[P_{k} f_{k}\right] \\
& \text { s.t. } \sum_{k=1}^{K} \mathbb{E}\left[P_{k} f_{k}\right] \leq Q_{\text {avg }} \\
& \sum_{k=1}^{K} \mathbb{E}\left[P_{k}\right] \leq P_{\text {avg }} \\
& \mathbb{E}\left[\int_{0}^{\infty} \int_{0}^{\infty} \ln (1\right. \\
& \left.+\frac{P_{k} g_{k}}{N_{0}+\rho h_{k}+\mathbb{E}\left[\sum_{j=1, j \neq k}^{K} P_{j} g_{j k}\right]}\right) \\
& \left.\times e^{-g_{k}} e^{-h_{k}} d g_{k} d h_{k}\right] \geq R_{\mathrm{avg}} \text { for } k=1, \ldots, K \text {. }
\end{aligned}
$$

The transmission power in the optimization problem (31a) and (31b) subject to (31c)-(31e) can be expressed as

$$
\begin{aligned}
P_{k}= & \int_{0}^{\infty} \int_{\frac{\rho h_{k}+A}{v+\xi_{k}}((1-v+\lambda)+\mu)}^{\infty}\left(\frac{v+\xi_{k}}{(1-v+\lambda)+\mu}\right. \\
& \left.-\frac{\rho h_{k}+A}{g_{k}}\right) e^{-g_{k}} e^{-h_{k}} d g_{k} d h_{k}
\end{aligned}
$$

$$
\begin{aligned}
= & \frac{e^{-\frac{(1-v+\lambda)+\mu}{v+\xi_{k}} A}}{\lambda}+(A+\rho) \operatorname{Ei}\left(-\left(\frac{(1-v+\lambda)+\mu}{v+\xi_{k}}\right) A\right) \\
& -e^{\frac{A}{\rho} \rho \operatorname{Ei}\left(-\frac{A+\left(\frac{(1-v+\lambda)+\mu}{v+\xi_{k}}\right) A \rho}{\rho}\right)}
\end{aligned}
$$

where $A=N_{0}+\mathbb{E}\left[\sum_{j=1, j \neq k}^{K} P_{j} g_{j k}\right]$. We note that (33) is a closed-form expression for the power at the $k$-th SU transmitter when no CSI is available at this SU transmitter.

In this case, we also use the iteration search based on the sub-gradient method as shown in equation (15)-(17) to find maximum ergodic sum rate by substituting (33) in (31a) which is expressed as

$$
\begin{aligned}
& C=\sum_{k=1}^{K} \int_{0}^{\infty} \int_{\left(\lambda^{(n+1)}+\mu^{(n+1)}\right)\left(\rho h_{k}+A\right)}^{\infty} \ln (1+ \\
& +\frac{g_{k}}{\rho h_{k}+A}\left(\frac{\left.e^{-\left(\frac{(1-v+\lambda(n+1))+\mu}{v+\xi_{k}^{(n+1)}}\right)}\right) A}{\lambda}+(A+\rho)\right. \\
& \times \operatorname{Ei}\left(-\left(\frac{\left(1-v+\lambda^{(n+1)}\right)+\mu^{(n+1)}}{v+\xi_{k}^{(n+1)}}\right) A\right) \\
& \left.-e^{\frac{A}{\rho}} \rho \operatorname{Ei}\left(-\frac{A+\left(\frac{\left(1-v+\lambda^{(n+1)}\right)+\mu^{(n+1)}}{v+\xi_{k}^{(n+1)}}\right) A \rho}{\rho}\right)\right)
\end{aligned}
$$

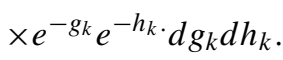

(34) gives the expression for the ergodic sum rate of the secondary links when the power at the $k$-th SU transmitter is kept constant.

\section{INTERFERENCE ACQUISITION}

We note that the practicality of the IE-optimal transmission method depends on the availability of the interference channel knowledge at the SU transmitter which could be challenging. Hence, here, we consider two different scenarios in terms of interference energy knowledge in the SU transmitter and investigate their IE performance.

\section{A. Imperfect CSI}

In this case, we consider a single cell scenario, hence no interference on the PU receiver is imposed from outside the cell. We then assume that the instantaneous information of the interference energy is known to the SU transmitters. Various strategies are proposed in the literature on how the information about CSI between secondary transmitters and primary receivers are obtained. For example, [41] considers a band manager that mediates between the primary and secondary users and provides the CSI information, or [13] proposes that the CSI can be directly fed back from the primary's receiver to the secondary user through an algorithm that allows the primary and secondary users to collaborate and exchange information. For primary networks that sublease their spectrum 
for monetary purposes, a level of cooperation between the primary and secondary networks can be expected. Moreover, our work does not require the phase information of the channel but requires only knowledge of the interference channel power gains. Hence, knowledge of the transmission signal power of the primary receiver is adequate to estimate the interference channel power gains [42]. In practice, certain cooperation in terms of parameter feedback between the PUs and the SUs is required.

Since obtaining a perfect interference energy information from all the neighbouring receivers could be challenging, in this scenario, we explain how the results of this paper can be used when the information of the interference channel, or equivalently, the information of the interference energy, is imperfect. The effects of imperfect interference CSI on the ergodic rate of CR systems is previously studied in [11] and [43]. Under imperfect CSI, it is assumed that the secondary transmitter performs minimum mean square error (MMSE) estimation of the interference channel gain. Here, we we adopt the following model:

$$
f_{k}=\varepsilon_{f_{k}}^{2} \hat{f}_{k}+\left(1-\varepsilon_{f_{k}}^{2}\right) \tilde{f}_{k},
$$

where $\hat{f}_{k}$ is the secondary channel MMSE estimation and $\tilde{f}_{k}$ is the estimation error which are independent of each other and also $\varepsilon_{f_{k}}^{2}$ represents the uncertainty with $0<\varepsilon_{f_{k}}^{2}<1$. The secondary transmitter, hence, has access to the estimated channel information along with the statistical information of the estimation error. Using (35), the MOP objective function (5b) changes into

$$
\min _{P_{k}} \sum_{k=1}^{K} \mathbb{E}\left[P_{k}\left(\varepsilon_{f_{k}}^{2} \hat{f}_{k}+\left(1-\varepsilon_{f_{k}}^{2}\right) \tilde{f}_{k}\right)\right] .
$$

Also, the MOP constraint $(5 \mathrm{c})$ changes into

$$
\text { s.t. } \sum_{k=1}^{K} \mathbb{E}\left[P_{k}\left(\varepsilon_{f_{k}}^{2} \hat{f}_{k}+\left(1-\varepsilon_{f_{k}}^{2}\right) \tilde{f}_{k}\right)\right] \leq Q_{\text {avg }} \text {. }
$$

Now, by applying the augmented Lagrangian, as introduced in (7 )-(10) the updated MOP when the CSI is imperfect can be solved.

\section{B. Multi-Cell Scenario}

In this case, we consider a multi-cell scenario wherein the instantaneous information about the interferences energy imposed on the PU receiver from the other cells is not available to the CR user. We also consider that there could be other operating CRNs within the same area. In this case aslo, we assume that the SU transmitter does not have instantaneous knowledge of the interference energy that the other CRNs impose on the PU receiver. Only the SU knows the total average interference power imposed on the PU receiver from the other networks (or other cells), referred to by $I_{0}$. Hence, the IE formula in this case becomes

$$
\eta_{\mathrm{IE}}=\frac{\sum_{k=1}^{K} \mathbb{E}\left[\ln \left(1+\mathrm{SINR}_{k}\right)\right]}{\sum_{k=1}^{K} \mathbb{E}\left[P_{k} f_{k}\right]+I_{0}} .
$$

We use the similar MOP solution approach as in Section III and convert it into a SOP. In this scenario, given that the value

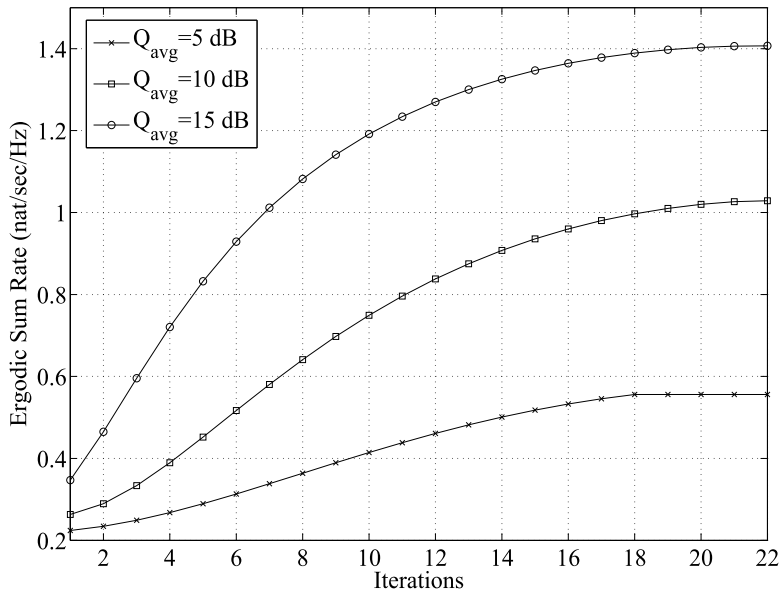

Fig. 2. Ergodic sum rate of secondary links versus the number of iterations with power control algorithm proposed in Table 1 with $K=2$ and $v=0.9$.

of $\left(I_{0}\right)$ which is added to the denominator of IE is constant, the developed solution method obtained in Section III applies to this scenario as well.

\section{NumericAl RESUlts}

In this section, we numerically evaluate the interference efficiency, maximum ergodic sum rate of secondary links and also the minimum total average interference power on PU receivers in Rayleigh fading channels. We further investigate the effects of varying weighting coefficient, $v$, fading severeness, the number of SU interferers, $K$, and having different CSI on the SU performance. In the following figures, we assume that $N_{0}=1, R_{\text {avg }}=2.5 \mathrm{nat} / \mathrm{sec} / \mathrm{Hz}$ and $P_{\text {avg }}=25 \mathrm{~dB}$, unless otherwise is indicated. Our simulation analysis suggest that $\sigma^{(0)} \in[1,5]$ works well in practice. In this case, the penalty parameter $\sigma^{(n)}$ would not be increased either too fast or too slowly avoiding ill-conditioning or reduction of the convergence rate. The subsequent values of $\sigma^{(n)}$ should be monotonically increased by $\sigma^{(n+1)}=\tau \sigma^{(n)}$ in which $\tau$ is a scalar with $\tau>1$. In our numerical analysis, we assume that $\tau=2$. Additional guidelines for choosing $\sigma^{(n)}$ and $\tau$ can be found in [30, Sec. 4.2].

In Fig. 2, the plots for the sum rate of secondary links of the CR network under the assumption of full CSI versus the number of iterations in the power control algorithm, proposed in Table 1, are given. In this figure, we assume that $K=2$ and $v=0.9$. The figure shows that after around 25 iterations, all three curves have converged to their corresponding optimal points.

Fig. 3 presents the plots for sum rate of multiple secondary links for different number of $K$ under full CSI versus the average interference power threshold $\left(Q_{\text {avg }}\right)$ with $v=0.9$. The figure reveals that as the number of secondary links increases, the ergodic sum rate decreases. For instance, when $Q_{\text {avg }}=10 \mathrm{~dB}$, the sum rate of two secondary links is almost 1.05 nats $/ \mathrm{s} / \mathrm{Hz}$ but the ergodic sum rate of four SU links is less than 0.9 nats $/ \mathrm{s} / \mathrm{Hz}$. Consequently, the secondary interferers significantly decrease the ergodic sum rate degrading the performance of the CRN. 


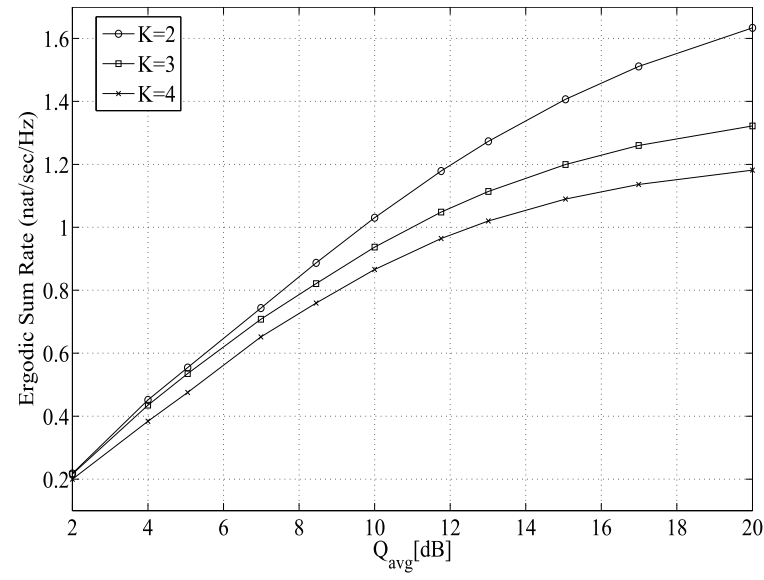

Fig. 3. Ergodic sum rate versus the interference power constraint limit for various number of the secondary transmitters under full CSI with $v=0.9$.

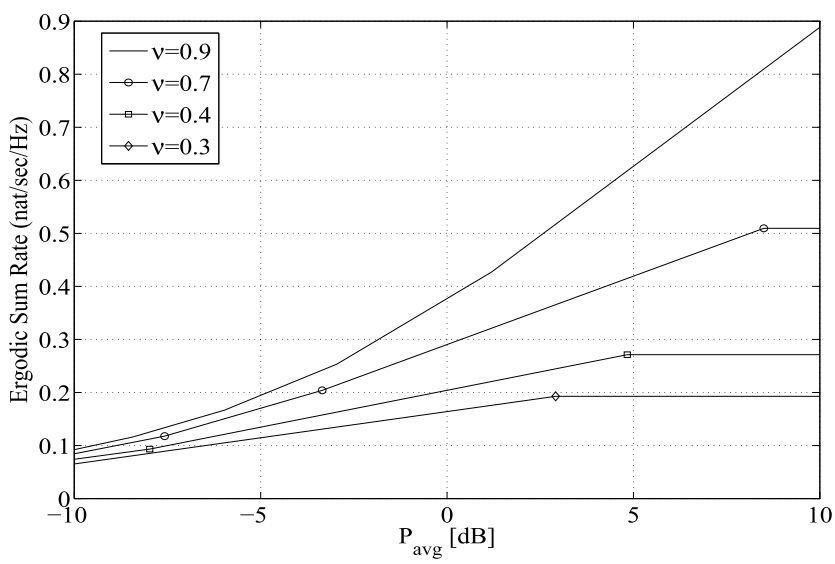

Fig. 4. Ergodic sum rate versus $P_{\text {avg }}$ for various weighting factor $v$ with $\rho=5 \mathrm{~dB}$ and $K=2$.

Fig. 4 displays the ergodic sum rate versus $P_{\text {avg }}$ for different values of weighting factor $v$ with $K=2$. As expected, the figure shows that by reducing the value of $v$ the ergodic sum rate also decreases. This happens because when the weighting factor increases, the system gives more importance to the sum rate.

The impact of varying weighting coefficient $(v)$ on the ergodic sum rate and the average interference power imposed on the PU receiver as a result of CR users transmission are respectively shown in Fig. 5 and 6. From Fig. 5 we observe that for $v$ between 0 and 0.5 , the ergodic sum rate is very low whereas for the higher value $v$ ergodic sum rate increases sharply with $v$. Fig. 6 shows that by increasing $v$ the minimum average interference power is also increasing.

Fig. 7 displays the maximum ergodic sum rate of secondary link against the minimum average interference power for various values of $v$ with $K=2$. This figure indicates a trade off between contradicting objectives that are the maximum sum rate against the minimum average interference power. In detail, we can see that higher capacity is achieved when higher interference is created. The rate, however, does not increase after the point where interference reaches a certain

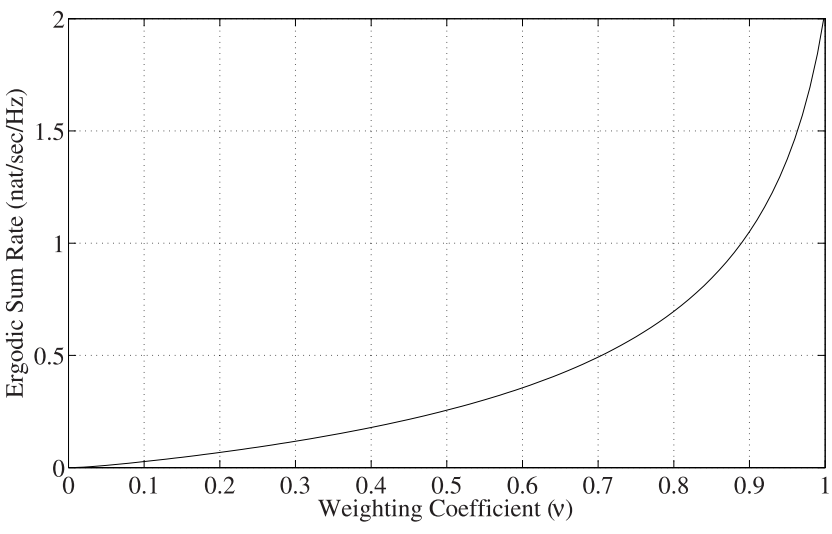

Fig. 5. Ergodic sum rate versus the weighting factor $v$ with $K=2$.

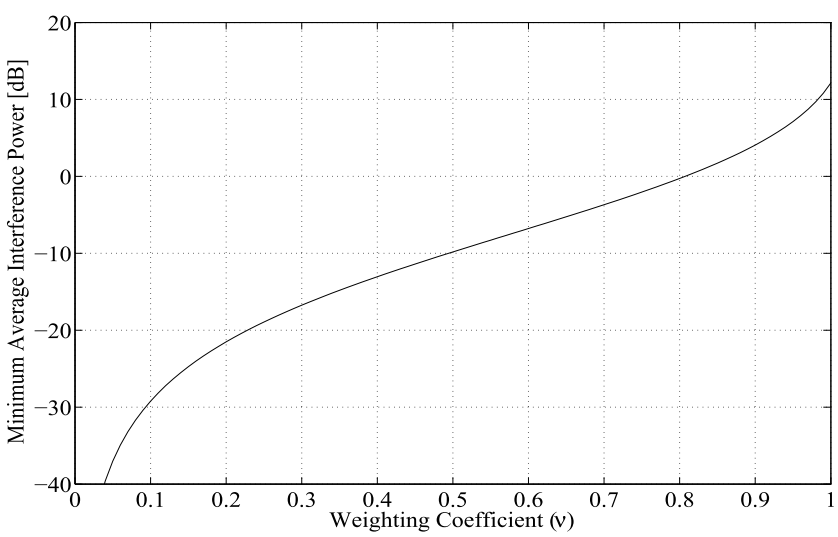

Fig. 6. Average interference power imposed on the PU receiver versus weighting factor $v$ with $K=2$.

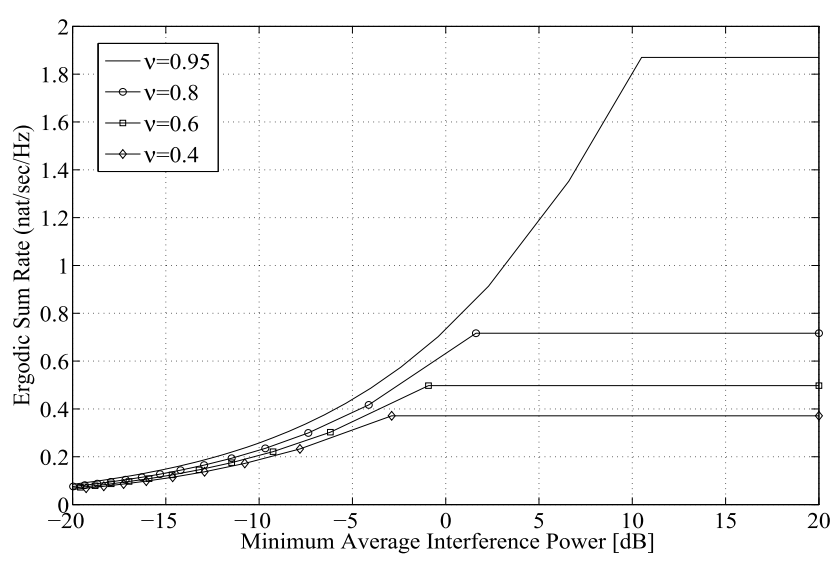

Fig. 7. Ergodic sum rate versus average interference power for various values of $v$ with $K=2$.

threshold. When the importance factor increases, the sum rate saturates at higher values of interference.

The effect of varying weighting coefficient $(v)$ on IE is shown in Fig. 8 which includes the plots for the IE versus the weighting factor when two SUs sharing the spectrum with a PU. This figure shows that the IE decreases monotonically with $v$. The slop of the plot however slows down after $v=0.1$. Hence, the figure reveals that the IE of the system is more sensitive to the weighting factor for smaller values of $v$. 


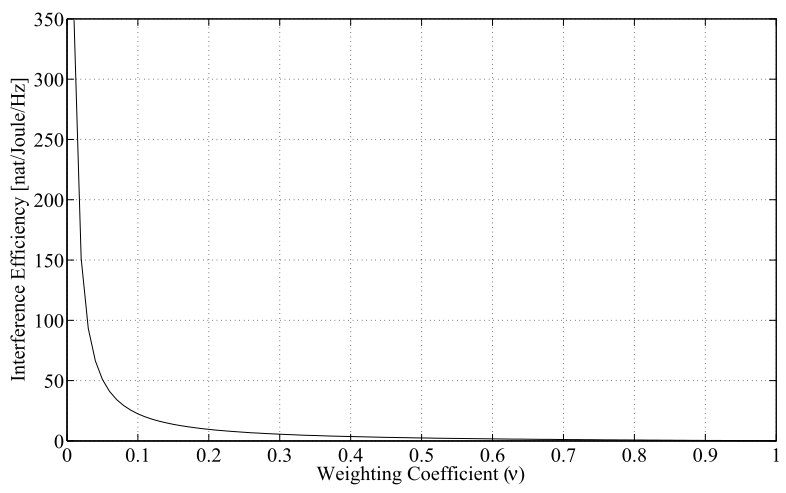

Fig. 8. Impact of varying $v$ on IE.

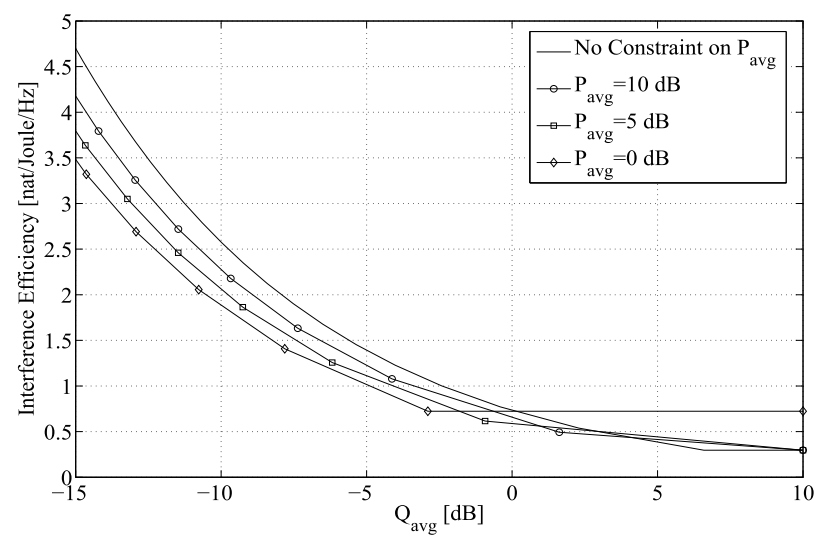

Fig. 9. IE versus $Q_{\text {avg }}$ for various values of $P_{\text {avg }}$.

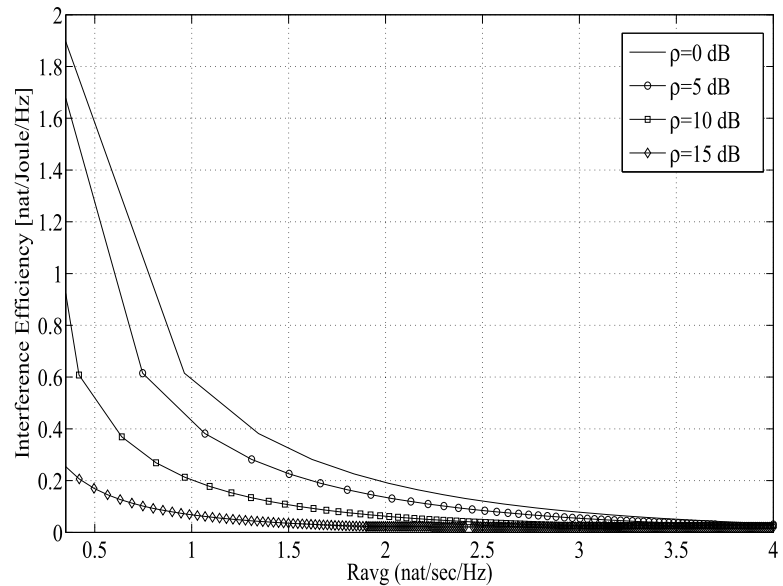

Fig. 10. IE versus $R_{\text {avg }}$ for various values of $\rho$.

Fig. 9 shows the plots for the IE of the CRN against $Q_{\text {avg }}$ for various values of $P_{\text {avg. }}$. In this figure, IE decreases when $Q_{\text {avg }}$ increases. The IE, however, remains constant after a certain threshold that depends on the value of $P_{\text {avg }}$.

The behaviour of the IE versus $R_{\text {avg }}$ for various values of the PU transmit power $(\rho)$ is shown in Fig. 10. From this figure, we can see that IE decreases when $R_{\text {avg }}$ increases. Also, for a fixed value of $R_{\text {avg }}$, IE decreases when $\rho$ increases.

Fig. 11 and Fig. 12 include the plots for the sum rate versus $Q_{\mathrm{avg}}$ under partial CSI for different values of $\rho$ with

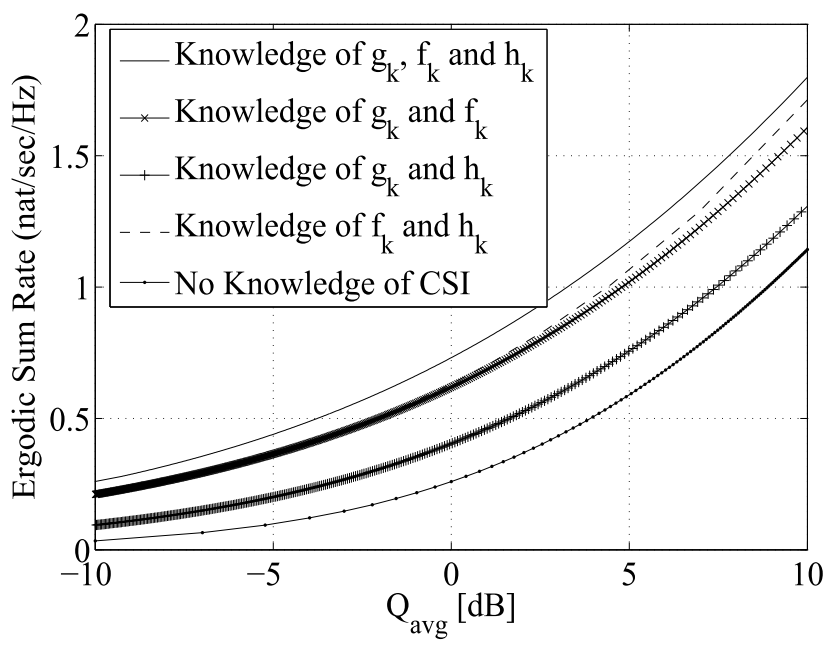

Fig. 11. Impact of reducing different CSI on the ergodic sum rate with $\rho=5 \mathrm{~dB}$.

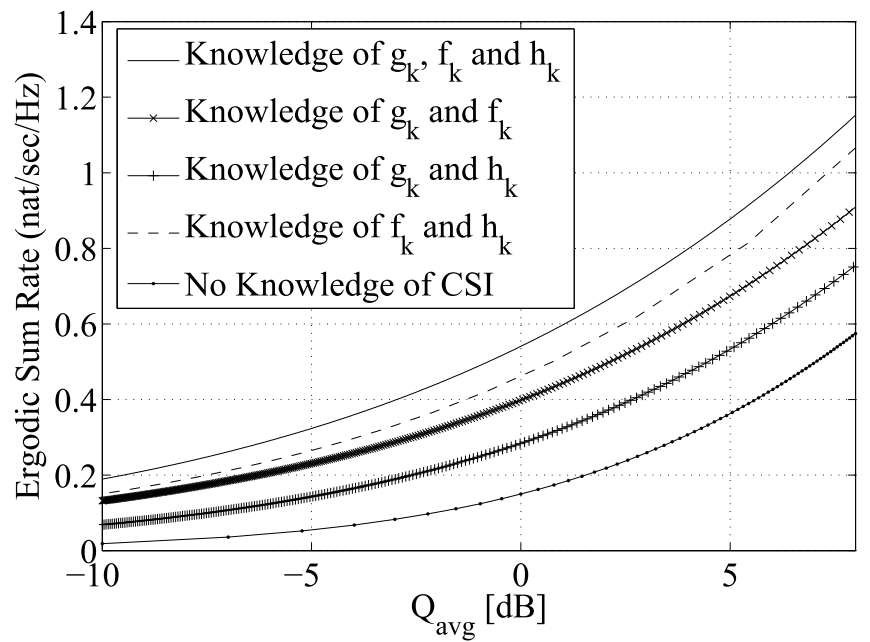

Fig. 12. Impact of reducing different CSI on the ergodic sum rate with $\rho=10 \mathrm{~dB}$.

$v=0.95$ and $A=1$. Comparing Fig. 11 with Fig. 12 indicates that the interference from the PU transmitter can have a big impact on the CRN ergodic sum rate. As we can see, the CRN ergodic sum rate in all cases increases with $Q_{\text {avg }}$. Further examination of Fig. 11 and Fig. 12 reveals that the highest sum rate occurs when instantaneous knowledge of $g_{k}, f_{k}$ and $h_{k}$ is available at the SU transmitter while the lowest sum rate occurs when no instantaneous knowledge of CSI is available at the SU transmitter. Another important observation is that there is a very small sum rate difference between the cases when the instantaneous knowledge of $g_{k}, f_{k}$ and $h_{k}$ are made available at the $k$ th SU transmitter and when the only knowledge of $f_{k}$ and $h_{k}$ are available at the $k$ th SU transmitter. Hence, implying that having side information of $g_{k}$ at the $k$ th SU transmitter has a small effect on the system performance. We further observe that when only CSI of $f_{k}$ is removed, the secondary link loses most of the sum rate advantage that can be achieved by having knowledge of $g_{k}, f_{k}$ and $h_{k}$. Therefore, $f_{k}$ has the highest impact on the sum rate of the system, while having $g_{k}$ has 


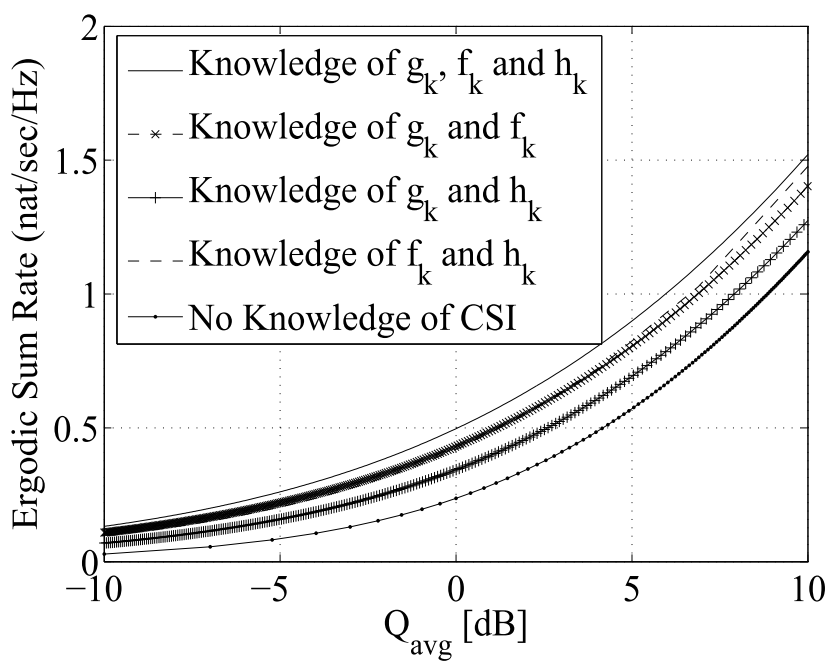

Fig. 13. Impact of reducing different CSI on the ergodic sum rate with $\rho=5 \mathrm{~dB}$ and $m=2$.

very minimal impact. Furthermore, the effect of reducing only $h_{k}$ is less than $f_{k}$ and bigger than $g_{k}$ such that by removing only the instantaneous knowledge of CSI $h_{k}$, the system loses almost half of the obtainable sum rate.

In addition, we also include the result of a Nakagami fading channel, where channel power gain $g_{k}, f_{k}$ and $h_{k}$ are distributed according to the following Gamma distribution

$$
f(x)=\frac{m^{m} x^{m-1}}{\Gamma(m)} e^{-m x},
$$

in Fig. 13 which shows ergodic sum rate against $Q_{\text {avg }}$ under the constraints on average interference power and total transmit power for $\rho=5 \mathrm{~dB}$ and $m=2$. The Nakagami parameter indicates the severity of fading, such that $m=1$ corresponds to Rayleigh fading and as $m=\infty$, the Nakagami fading channel converges to a non-fading AWGN channel [12]. Comparing Fig. 13 and Fig. 11 reveals that as severity parameter $m$ increases the difference between the highest and lowest ergodic sum rate of the SU link decreases.

\section{A. Interference Acquisition}

Fig. 14 shows IE versus $\eta$ for different values of $I_{0}$. As can be seen in this figure, the maximum achievable IE decreases with $I_{0}$. Also as $I_{0}$ increases, the $\eta$ at which the IE is maximized increases. The point at which IE is maximized is when the optimal value of the SOP (equation (6)) is equal to zero. We note that by considering $I_{0}$ in (6), this equation is expressed as

$$
\begin{array}{rl}
\max _{P_{k}} & v\left(\sum_{k=1}^{K} \mathbb{E}\left[\ln \left(1+\operatorname{SINR}_{k}\right)\right]\right) \\
& -(1-v)\left(\sum_{k=1}^{K} \mathbb{E}\left[P_{k} f_{k}\right]+I_{0}\right) .
\end{array}
$$

In Fig. 15, we have plotted equation (6) versus $\eta$. According to (44) in Appendix A, $\eta$ that returns the root for (6) is the $\eta$ that corresponds to the maximum IE. So, when each

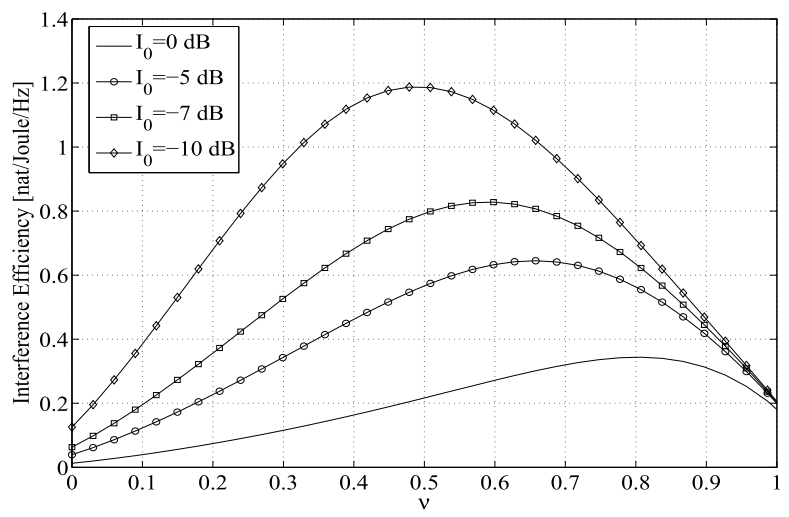

Fig. 14. IE versus $v$ for various values of $I_{0}$.

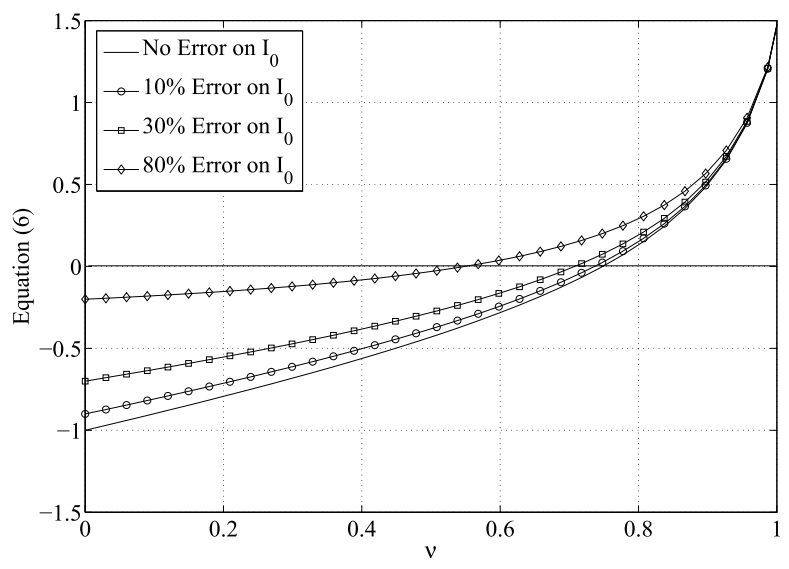

Fig. 15. Equation (6) versus $v$ with $I_{0}=0 \mathrm{~dB}$ and various values of error on $I_{0}$.

plot crosses the zero line, the corresponding optimal $\eta$ can be found. For example, when perfect knowledge of $I_{0}$ is available, the optimal $\eta$ is $\eta=0.765$. Now, by referring to Fig. 14, one can find the maximum IE, which is at $\eta=0.765$. In Fig. 15, we also include the plots when there are some error in the knowledge of $I_{0}$ at the SU transmitter. We note that when there is error in the information of $I_{0}$, the $v$ at which the plotted curve crosses the zero line departs from its optimal value at $\eta=0.765$. This, in turn, affects the IE-optimal transmission strategy and results in a lower achievable IE.

\section{B. IE Versus EE}

In Fig. 18, Fig. 16 and Fig. 17, we includes the results of two different adaptive power transmission schemes, namely 1) an IE-efficient approach: a power allocation scheme that maximizes the IE of the CRN (according to the derivations of this paper), and 2) an EE-efficient approach: a power allocation scheme that maximizes the energy efficiency of the SU network, which is defined as

$$
\mathrm{EE}=\frac{\sum_{k=1}^{K} \mathbb{E}\left[\ln \left(1+\mathrm{SINR}_{k}\right)\right]}{\sum_{k=1}^{K} \mathbb{E}\left[P_{k}\right]+P_{\mathrm{c}}},
$$

where $P_{\mathrm{c}}$ is a constant value of the circuit power consumption [44], [45]. Through these figures we can observe the 


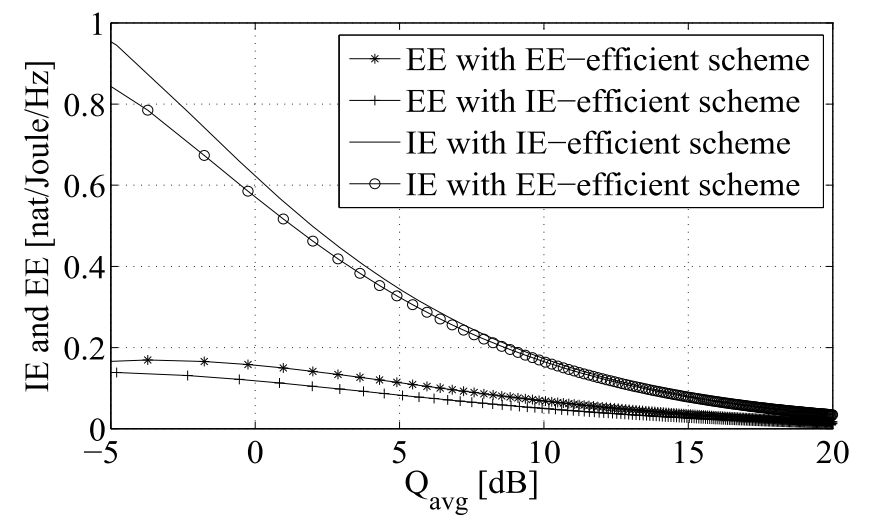

Fig. 16. IE and EE versus $Q_{\text {avg }}$ with $I_{0}=P_{\mathrm{c}}=-10 \mathrm{~dB}$.

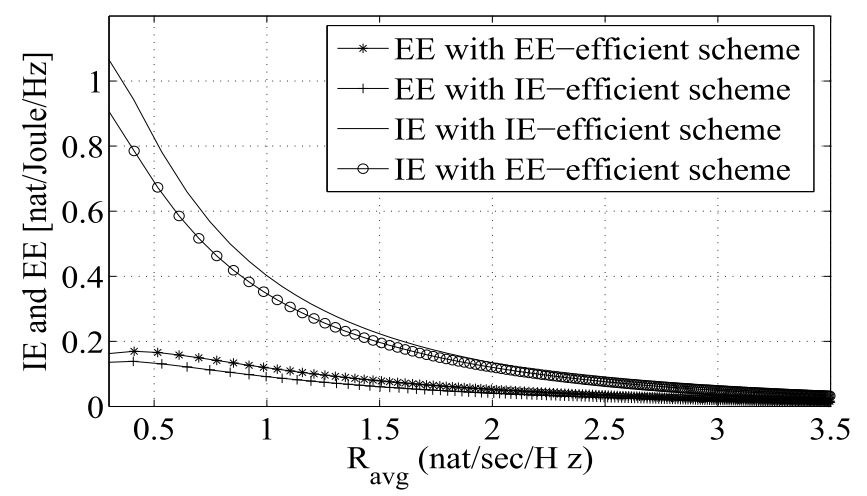

Fig. 17. IE and EE versus $R_{\text {avg }}$ with $I_{0}=P_{\mathrm{c}}=-10 \mathrm{~dB}$.

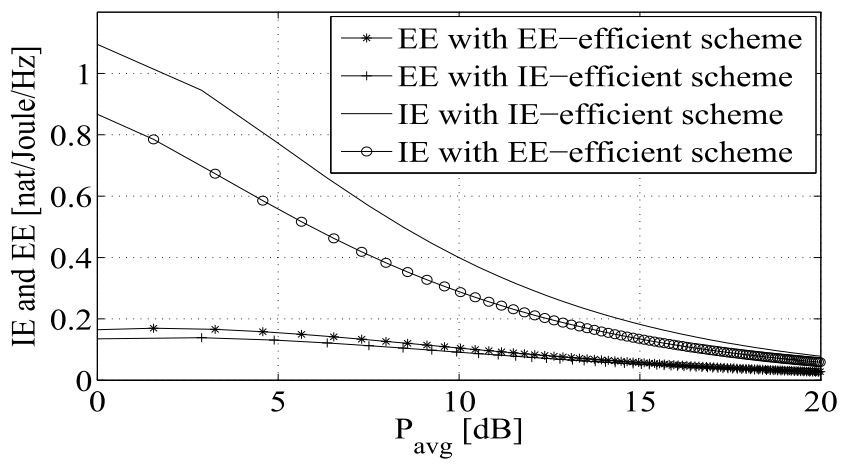

Fig. 18. IE and EE versus $P_{\text {avg }}$ with $I_{0}=P_{\mathrm{c}}=-10 \mathrm{~dB}$.

difference between the maximum achievable IE and EE under these two power allocation schemes.

In detail, Fig. 18 includes the plots for the maximum achievable IE and EE versus the $P_{\text {avg }}$ under IE-efficient and EE-efficient power allocation schemes when $I_{0}=-10 \mathrm{~dB}$, $P_{\mathrm{c}}=0 \mathrm{~dB}$ and $\rho=5 \mathrm{~dB}$. The figure shows that at low $\mathrm{SU}$ transmit power limits, i.e., $P_{\text {avg }} \leq 5 \mathrm{~dB}$, although the achievable EE by the two schemes are not very different, but the achievable IE by the IE-efficient transmission scheme is much higher than the one with the EE-efficient scheme. This happens since the distribution of the power in the IE-efficient scheme is adopted to both direct and interference channels in the CRN, and hence, it can benefit from the variations of both channels. As the value for $P_{\text {avg }}$ increases, the IE that can be achieved by the two schemes become similar. The maximum achievable IE and EE versus $Q_{\text {avg }}$ and also versus $R_{\text {avg }}$ under these two transmission schemes are given in Fig. 16 and Fig. 17, respectively. The two figures show that at higher values of $Q_{\text {avg }}$ and $R_{\text {avg }}$, the performance of the two transmission schemes becomes similar.

\section{SUMMARY}

In this paper, we considered a spectrum-sharing system where multiple secondary links share the spectrum with an existing primary link. We have proposed a new performance factor called interference efficiency to analyze the sum rate of the CRN versus the imposed interference power on the PU receiver. By formulating the performance objective as an MOP which jointly maximizes the ergodic sum rate of the $\mathrm{CRN}$ and minimizes the average interference power on PU receiver, subject to average constraints on the interference power, total transmit power and minimum ergodic rates for the SUs, we aim to maximize the IE of the CRN. The MOP is converted into an SOP through weighted sum method. Numerical analysis indicate that the maximum rate highly depends on the weighting coefficient by which the competing objective functions are linearly combined into a single objective function. Furthermore, we investigated the effect of different levels of CSI, which has been provided to each $\mathrm{SU}$ transmitter, on the performance of the system. From the numerical result, we observed that the sum rate of the CRN highly depends on having side information of the links between the SU transmitter and the PU receiver at each SU transmitter. However, the side information of the link between the SU transmitter and the SU receiver at the SU transmitter has negligible impact on its rate. Another important result is that the higher the number of secondary interferers results in achieving a lower ergodic sum rate.

\section{APPENDIX A}

In order to give a formal proof of Lemma 1, we have employed a general fractional programming which can be formulated as

$$
\max \theta(x)=\frac{f(x)}{g(x)}: x \in X,
$$

where $X$ is a nonempty compact of $R^{n} . f(x)$ and $g(x)$ are continuous real-valued functions of $x \in X$ and $g(x)>0$ for all $x \in X$. Let define

$$
H\left(q^{*}\right)=\max _{x}\left\{f(x)-q^{*} g(x): x \in X\right\},
$$

as the minimum value of $f(x)-q g(x)$ with each fixed $q^{*}$.

It is proved in [38] that

$$
q^{*}=\frac{f\left(x^{*}\right)}{g\left(x^{*}\right)}=\max _{x}\left\{\frac{f(x)}{g(x)}: x \in X\right\},
$$

if and only if

$$
H\left(q^{*}\right)=H\left(q^{*}, x^{*}\right)=\max _{x}\left\{f(x)-q^{*} g(x): x \in X\right\}=0 .
$$


Therefore, from (43) and (44), it can be found that the optimal solution $x$ of (41) is the optimal solution of (42) when (44) holds.

On the other hand, we now formulate a general MOP with two objectives as follows:

$$
\begin{aligned}
& \max f(x) \\
& \min g(x) \\
& \text { s.t. } x>0
\end{aligned}
$$

In MOPs, it is characteristic that no unique solution exists but a set of mathematically equally good solutions can be identified. These solutions are known as Pareto Optimal solutions. With different parameters for the scalarization, different Pareto Optimal solutions are produced. A solution is called nondominated or Pareto Optimal if none of the objective functions can be improved in value without degrading some of the other objective values. By combining the competing objective functions (45) and (46) into a single objective function linearly through weighting coefficients, the objective functions in the MOP can be changed into a SOP as follows

$$
\max f(x)-v g(x) .
$$

By comparing (48), (44) and (41), one can show that optimal set of (48) is inclusive of the solution for (41). The value of $v$ that makes the maximum of the SOP equivalent to zero will yield a solution for the fractional programming problem.

We note that (48) is equal to (6) when one replaces $f(x)$ with ergodic sum rate and $g(x)$ with interference power. The solution of the MOP in (6) subject to (4b)-(4d) is a pareto optimal region. There is a point on this region that returns the solution of the IE maximization in (4). To find this point, we need to find the value of $v$ that makes (6) equal to zero.

Finally, we note that the MOP can be applied to find the optimal solution from $n$ objective functions ( $n$ can be bigger than 2) whereas the fractional programming only works for two functions.

\section{APPENDIX B}

If we consider the following general concave maximization over a set as:

$$
\begin{aligned}
& \max f(\mathbf{x}) \\
& \text { s.t. } \mathbf{x} \in \chi .
\end{aligned}
$$

This subgradient method generates a sequence of feasible points as

$$
\mathbf{x}(t+1)=[\mathbf{x}(t)+\alpha(t) \mathbf{s}(t)]_{\chi}
$$

where $\mathbf{s}(t)$ denotes a gradient of $f$ evaluated at the point $\mathbf{x}(t)$ if $f$ is differentiable, $[.]_{\chi}$ is the projection onto the feasible set $\chi$, and $\alpha(t)$ represents a positive step-size. The distance of the current solution $\mathbf{x}(t)$ to the optimal solution $\mathbf{x}^{*}$ for sufficiently small step-size decreases which makes the subgradient method converge. With different choices of stepsizes, many results on convergence of the subgradient method can be found [39], [40]. For example, for a diminishing stepsize rule $\alpha(t)=\frac{1+m}{t+m}$, in which $m$ denotes a fixed nonnegative number, it is guaranteed that the algorithm converges to the optimal value [40]. For a constant step-size $\alpha(t)=\alpha$, which is more convenient for distributed algorithms, the subgradient algorithm converges to the best value within some range of the optimal value provided that the step-size is sufficiently small [39].

\section{REFERENCES}

[1] Promoting More Efficient Use of Spectrum through Dynamic Spectrum Use Technologies, document ET Docket nos. 10-237, Federal Commun. Commission, Washington, DC, USA, Nov. 2010.

[2] E. Z. Tragos, S. Zeadally, A. G. Fragkiadakis, and V. A. Siris, "Spectrum assignment in cognitive radio networks: A comprehensive survey," IEEE Commun. Surveys Tut., vol. 15, no. 3, pp. 1108-1135, 3rd Quart., 2013.

[3] G. I. Tsiropoulos, O. A. Dobre, M. H. Ahmed, and K. E. Baddour, "Radio resource allocation techniques for efficient spectrum access in cognitive radio networks," IEEE Commun. Surveys Tut., vol. 18, no. 1, pp. 824-847, 1st Quart., 2016.

[4] K. Arshad et al., "Resource management for QoS support in cognitive radio networks," IEEE Commun. Mag., vol. 52, no. 3, pp. 114-120, Mar. 2014.

[5] P. Madhusudhanan, J. G. Restrepo, Y. Liu, T. X. Brown, and K. R. Baker, "Downlink performance analysis for a generalized shotgun cellular system," IEEE Trans. Wireless Commun., vol. 13, no. 12, pp. 6684-6696, Dec. 2014.

[6] W. Zhong and J. Wang, "Energy efficient spectrum sharing strategy selection for cognitive MIMO interference channels," IEEE Trans. Signal Process., vol. 61, no. 14, pp. 3705-3717, Jul. 2013.

[7] W. Zhong, G. Chen, S. Jin, and K.-K. Wong, "Relay selection and discrete power control for cognitive relay networks via potential game," IEEE Trans. Signal Process., vol. 62, no. 20, pp. 5411-5424, Oct. 2014.

[8] M. M. Mahyari, A. Shojaeifard, and M. Shikh-Bahaei, "Probabilistic radio resource allocation over CDMA-based cognitive radio networks," IEEE Trans. Veh. Technol., vol. 64, no. 8, pp. 3560-3565, Aug. 2015.

[9] L. Sboui, Z. Rezki, and M.-S. Alouini, "Achievable rate of spectrum sharing cognitive radio systems over fading channels at lowpower regime," IEEE Trans. Wireless Commun., vol. 13, no. 11, pp. 6461-6473, Nov. 2014.

[10] P. J. Smith, P. A. Dmochowski, H. A. Suraweera, and M. Shafi, "The effects of limited channel knowledge on cognitive radio system capacity," IEEE Trans. Veh. Technol., vol. 62, no. 2, pp. 927-933, Feb. 2013.

[11] L. Sboui, Z. Rezki, and M.-S. Alouini, "A unified framework for the ergodic capacity of spectrum sharing cognitive radio systems," IEEE Trans. Wireless Commun., vol. 12, no. 2, pp. 877-887, Feb. 2013.

[12] A. J. Goldsmith and P. P. Varaiya, "Capacity of fading channels with channel side information," IEEE Trans. Inf. Theory, vol. 43, no. 6, pp. 1986-1992, Nov. 1997.

[13] A. Jovicic and P. Viswanath, "Cognitive radio: An information-theoretic perspective," IEEE Trans. Inf. Theory, vol. 55, no. 9, pp. 3945-3958, Sep. 2009.

[14] M. R. Mili and K. A. Hamdi, "The effect of different levels of side information on the ergodic capacity in cognitive radio networks," in Proc. GLOBECOM, Dec. 2014, pp. 1090-1095.

[15] L. Musavian and S. Aissa, "Fundamental capacity limits of cognitive radio in fading environements with imperfect channel information," IEEE Trans. Commun., vol. 57, no. 11, pp. 3472-3480, Nov. 2009.

[16] Z. Rezki and M. S. Alouini, "Ergodic capacity of cognitive radio under imperfect channel-state information," IEEE Trans. Veh. Commun., vol. 61, no. 5, pp. 2108-2119, Jun. 2012.

[17] X. Gong, A. Ispas, G. Dartmann, and G. Ascheid, "Power allocation and performance analysis in spectrum sharing systems with statistical CSI," IEEE Trans. Wireless Commun., vol. 12, no. 4, pp. 1819-1831, Apr. 2013

[18] M. Gastpar, "On capacity under receive and spatial spectrum-sharing constraints," IEEE Trans. Inf. Theory, vol. 53, no. 2, pp. 471-487, Feb. 2007.

[19] J. Tang, D. K. C. So, E. Alsusa, and K. A. Hamdi, "Resource efficiency: A new paradigm on energy efficiency and spectral efficiency tradeoff," IEEE Trans. Wireless Commun., vol. 13, no. 8, pp. 4656-4669, Aug. 2014.

[20] Z. Song, Q. Ni, K. Navaie, S. Hou, and S. Wu, "Energy- and spectralefficiency tradeoff with $\alpha$-fairness in downlink OFDMA systems," IEEE Commun. Lett., vol. 19, no. 7, pp. 1265-1268, Jul. 2015. 
[21] H. Pervaiz, L. Musavian, N. Qiang, and Z. Ding, "Energy and spectrum efficient transmission techniques under QoS constraints toward green heterogeneous networks," IEEE Access, vol. 3, pp. 1655-1671, 2015.

[22] K. Miettinen, Nonlinear Multiobjective Optimization. Springer, 1999.

[23] E. Bedeer, O. A. Dobre, M. H. Ahmed, and K. E. Baddour, "Joint optimization of bit and power loading for multicarrier systems," IEEE Wireless Commun. Lett., vol. 2, no. 4, pp. 447-450, Aug. 2013

[24] E. Bedeer, O. A. Dobre, M. H. Ahmed, and K. E. Baddour, "A multiobjective optimization approach for optimal link adaptation of OFDMbased cognitive radio systems with imperfect spectrum sensing," IEEE Trans. Wireless Commun., vol. 13, no. 4, pp. 2339-2351, Apr. 2014.

[25] X. Hong, C. Zheng, J. Wang, J. Shi, and C. X. Wang, "Optimal resource allocation and EE-SE trade-off in hybrid Cognitive Gaussian relay channels," IEEE Trans. Wireless Commun., vol. 14, no. 8, pp. 4170-4181, Aug. 2015

[26] W. Shi, S. Wang, and D. Chen, "Energy- and spectrum-efficiency tradeoff in OFDM-based cognitive radio systems," in Proc. GLOBECOM, Dec. 2014, pp. 3092-3097.

[27] D. W. K. Ng, E. S. Lo, and R. Schober, "Multiobjective resource allocation for secure communication in cognitive radio networks with wireless information and power transfer," IEEE Trans. Veh. Technol., vol. 65, no. 5, pp. 3166-3184, May 2016.

[28] M. R. Mili, L. Musavian, K. A. Hamdi, and F. Marvasti, "How to increase energy efficiency in cognitive radio networks," IEEE Trans. Commun., vol. 64, no. 5, pp. 1829-1843, May 2016.

[29] Z.-Q. Luo and S. Zhang, "Dynamic spectrum management: Complexity and duality," IEEE J. Sel. Topics signal Process., vol. 2, no. 1, pp. 57-73, Feb. 2008.

[30] D. P. Berteskas, Nonlinear Programming, 2nd ed. Cambridge, MA, USA: MIT Press, 1999

[31] J. Nocedal and S. J. Wright. Numerical Optimization. New York, NY, USA: Springer, 2006

[32] D. P. Bertsekas, "On penalty and multiplier methods for constrained minimization," SIAM J. Control Optim., vol. 14, no. 2, pp. 216-235, 1976.

[33] D. P. Bertsekas, Constrained Optimization and Lagrange Multiplier Methods. New York, NY, USA: Academic, 1982.

[34] S. Boyd and L. Vandenberghe, Convex Optimization. Cambridge, U.K.: Cambridge Univ. Press, 2004

[35] W. Yu and R. Lui, "Dual methods for nonconvex spectrum optimization of multicarrier systems," IEEE Trans. Commun., vol. 54, no. 7, pp. 1310-1322, Jul. 2006.

[36] D. P. Bertsekas, Convex Optimization Algorithms. Belmont, MA, USA: Athena Scientific, 2015

[37] I. S. Gradshteyn and I. M. Ryzhik, Table of Integrals, Series, and Products, 7th ed. San Diago, CA: Academic, 2007.

[38] W. Dinkelbach, "On nonlinear fractional programming," Manage. Sci., vol. 13 , no. 7, pp. 492-498, 1967.

[39] D. P. Bertsekas, Nonlinear Programming, 2nd ed. Belmont, MA, USA: Athena Scientific, 1999

[40] D. P. Bertsekas, A. Nedic, and A. E. Ozdaglar, Convex Analysis and Optimization. Belmont, MA, USA: Athena Scientific, 2003.

[41] J. M. Peha, "Approaches to spectrum sharing," IEEE Commun. Mag., vol. 43, no. 2, pp. 10-12, Feb. 2005.
[42] L. Musavian, S. Aïssa, and S. Lambotharan, "Effective capacity for interference and delay constrained cognitive radio relay channels," IEEE Trans. Wireless Commun., vol. 9, no. 5, pp. 1698-1707, May 2010.

[43] N. Mokari, S. Parsaeefard, P. Azmi, H. Saeedi, and E. Hossain, "Robust ergodic uplink resource allocation in underlay OFDMA cognitive radio networks," IEEE Trans. Mobile Comput., vol. 15, no. 2, pp. 419-431, Feb. 2016.

[44] E. Bedeer, O. Amin, O. A. Dobre, M. H. Ahmed, and K. E. Baddour, "Energy-efficient power loading for OFDM-based cognitive radio systems with channel uncertainties," IEEE Trans. Veh. Technol., vol. 64, no. 6, pp. 2672-2677, Jun. 2015.

[45] M. Peng, K. Zhang, J. Jiang, J. Wang, and W. Wang, "Energyefficient resource assignment and power allocation in heterogeneous cloud radio access networks," IEEE Trans. Veh. Technol., vol. 64, no. 11, pp. 5275-5287, Nov. 2015

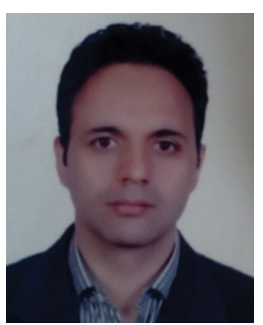

Mohammad Robat Mili received the Ph.D. degree in electrical and electronic engineering from the University of Manchester, U.K., in 2012. He joined Sharif University of Technology, Iran, as a PostDoctoral Fellow. His primary research interests include wireless and mobile communications networks, cognitive radio systems, ad hoc networks, green communication, D2D communication, and applied mathematical methods, such as optimization and game theory.

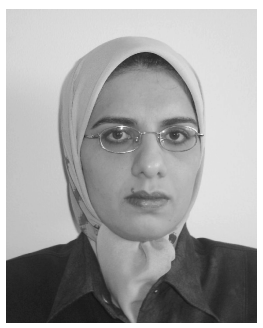

Leila Musavian (S'05-M'07) received the Ph.D. degree in Telecommunications from Kings College London, U.K., in 2006. She was a Research Associate with McGill University from 2011 to 2012 and a Post-Doctoral Fellow with INRS-EMT, Canada, from 2006 to 2008. She was a Lecturer with InfoLab21, Lancaster University, from 2012 to 2016. She is currently a Reader with the School of Computer Science and Electronics Engineering, University of Essex. Her research interests include radio resource management for next generation wireless networks, CRNs, energy harvesting, green communication, energy-efficient transmission techniques, cross-layer design for delay QoS provisioning, and 5G systems. She is an Editor of the IEEE TRANSACTIONS ON WIRELESS COMMUNICATIONS and an Executive Editor of Wiley's Transactions on Emerging Telecommunications Technologies. 